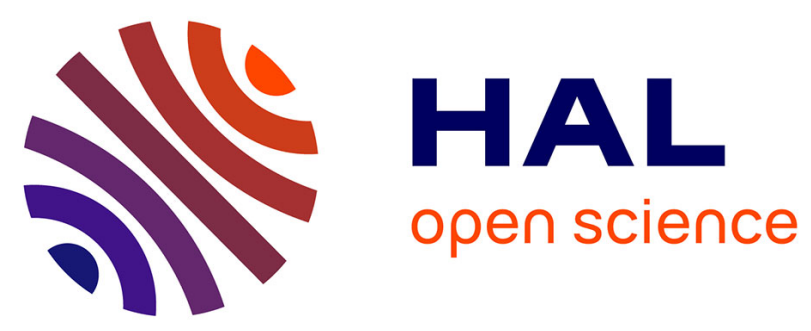

\title{
STRONG LOCAL MINIMIZERS IN OPTIMAL CONTROL PROBLEMS WITH STATE CONSTRAINTS: SECOND-ORDER NECESSARY CONDITIONS
}

Hélène Frankowska, Nikolai P. Osmolovskii

\section{To cite this version:}

Hélène Frankowska, Nikolai P. Osmolovskii. STRONG LOCAL MINIMIZERS IN OPTIMAL CONTROL PROBLEMS WITH STATE CONSTRAINTS: SECOND-ORDER NECESSARY CONDI-

TIONS. SIAM Journal on Control and Optimization, 2018, 56, pp.2353-2376. hal-02126111

HAL Id: hal-02126111

https://hal.science/hal-02126111

Submitted on 10 May 2019

HAL is a multi-disciplinary open access archive for the deposit and dissemination of scientific research documents, whether they are published or not. The documents may come from teaching and research institutions in France or abroad, or from public or private research centers.
L'archive ouverte pluridisciplinaire HAL, est destinée au dépôt et à la diffusion de documents scientifiques de niveau recherche, publiés ou non, émanant des établissements d'enseignement et de recherche français ou étrangers, des laboratoires publics ou privés. 


\title{
STRONG LOCAL MINIMIZERS IN OPTIMAL CONTROL PROBLEMS WITH STATE CONSTRAINTS: SECOND-ORDER NECESSARY CONDITIONS
}

\author{
HÉLÈNE FRANKOWSKA* AND NIKOLAI P. OSMOLOVSKII ${ }^{\dagger}$
}

\begin{abstract}
This paper is devoted to second-order necessary optimality conditions for strong local minima for a Mayer type optimal control problem with a general control constraint $U \subset \mathbb{R}^{m}$, and state and final-point constraints described by a finite number of inequalities. We use the second order linearization of a relaxed differential inclusion associated to the control system to find a convex subset of second order tangents to the set of its trajectories. This leads to second-order necessary optimality conditions via a straightforward way, based on separation theorems.
\end{abstract}

Key words. Optimal control, state constraints, strong local minimum, maximum principle, second-order necessary conditions, second order tangents, differential inclusion.

AMS subject classifications. Primary 49K15; Secondary 34H05, 49K24.

1. Introduction. Consider the Mayer optimal control problem

$$
\begin{gathered}
\text { Minimize } J(x, u):=g_{0}(x(1)) \\
g_{i}(x(1)) \leq 0, \quad i=1, \ldots, k, \\
\dot{x}(t)=f(x(t), u(t)), \quad x(0)=x_{0}, \quad u(t) \in U \quad \text { a.e. in }[0,1],
\end{gathered}
$$

where $U$ is an arbitrary compact subset of $\mathbb{R}^{m}, x_{0} \in \mathbb{R}^{n}$ is given and functions $g_{i}: \mathbb{R}^{n} \rightarrow \mathbb{R}, f: \mathbb{R}^{n} \times \mathbb{R}^{m} \rightarrow \mathbb{R}^{n}, \Phi_{j}: \mathbb{R}^{n} \rightarrow \mathbb{R}$ are assumed to be twice continuously differentiable. Even though the approach developed in this work can be applied to more general initial point constraint, non autonomous control systems and time dependent control constraint $u(t) \in U(t)$ with possibly unbounded sets $U(t)$, to keep the presentation less technical we do not seek here the most general setting.

A vast literature is devoted to necessary optimality conditions, because many applied models do involve optimisation and control systems. Due to the appearance of state and control constraints in these models, such framework was considered by many authors. The "pure state constraint" (involving the state variable only) being particularly difficult to approach, some authors restricted their attention to the so

${ }^{*}$ CNRS, IMJ-PRG, Sorbonne Université, case 247, 4 place Jussieu, 75252 Paris, France. The research of this author benefited from the support of the FMJH Program Gaspard Monge in optimization and operation research, and from the support to this program from EDF under the grant PGMO 2015-2832H. E-mail: helene.frankowska@imj-prg.fr

${ }^{\dagger}$ University of Technology and Humanities, 26-600 Radom, ul. Malczewskiego 20A, Poland; Systems Research Institute, Polish Academy of Sciences, ul. Newelska 6, 01-447, Warszawa, Poland; Moscow State University of Civil Engineering, Jaroslavskoe shosse 26, Moscow, Russia. The research of this author is supported by the Russian Foundation for Basic Research under grant 16-01-00585 E-mail: osmolovski@uph.edu.pl 
called "finite order pure state constraints". Then, under several structural assumptions, the control problem at hand can be transformed into the one involving the mixed state-control constraints only, where the situation is rather well understood.

The first results on the maximum principle for "pure" state constraints like (1.4) go back to Gamkrelidze [11], where it was assumed that the optimal process has a special structure. Dubovitskii and Milyutin [6] avoided such restrictive requirements, introducing measures in the definition of costate, leading to an exhaustive form of the maximum principle that is used in nowadays. The first-order conditions are well understood even when data are nonsmooth and were generalized in various ways in thousands of publications (including PDE and stochastic frameworks). In the deterministic control, these necessary conditions were derived for general control sets $U$ and merely measurable optimal controls. Let us underline that the existence results in optimal control of nonlinear systems do not guarantee any regularity of optimal controls, but only their measurability. Quite strong additional assumptions have to be imposed to get even continuity (or piecewise continuity) of optimal controls. The interested reader can find in [12] historical comments and a large bibliography of the earlier literature on the first and second-order optimality conditions under state constraints. We also refer to [15] for extended discussions on the constrained maximum principle in the deterministic case and further references to the earlier Russian literature on the subject, and to [23] for further developments in the nonsmooth framework.

In contrast with the first-order conditions, when working with second-order conditions, most of the authors suppose that optimal controls are continuous, or piecewise continuous, see for instance $[22,18,3]$ and the references therein. In addition, structural assumptions and constraint qualifications on optimal trajectory-control pairs are often imposed. For instance, in [3] weak local minimizers for an optimal control problem under finite order state constraints are discussed, assuming the Robinson constraint qualification and that the optimal trajectory has only finitely many boundary arcs. Let us recall that it was observed in [19] that, even for a smooth calculus of variations problem under a very simple state constraint and having a unique optimal trajectory, the transitions from the interior to the boundary of state constraints may occur an infinite number of times. More rarely, articles deal with measurable optimal controls and general control sets, as for instance [21] and [13]. In [21], using the time transformation introduced in [5], a second-order necessary condition for strong local minima is derived in a very general setting. However this condition addresses only a very narrow set of second order variations of controls, because only time transformations of the optimal control are taken into consideration in the second-order conditions. In [13] second-order conditions for weak minima are derived for an optimal control problem not involving the end point constraint using second order tangents to the control set $U$. In this last paper an "interiority" assumption is imposed on the solution set of a second-order linearized system, see also [14] for a further discussion of this assumption.

The approach we use here has its roots in $[13,8]$, where controls are assumed to be merely measurable and second order tangents to the set $U$ are used to state secondorder optimality conditions. This allows to work with very general control constraints which, in particular, imply known results when $U$ is given by inequality and equality type constraints (here we only discuss the case of constraints given by inequalities). Our paper differs however from $[13,14]$, where only weak minima are investigated, the final-state constraint is absent, and "interiority" assumption is imposed, while [8] requests the strong normal maximum principle to hold true. Also, in the difference 
with [8], we state second-order conditions involving only the critical cone and second order tangents to the set $U$, instead of using trajectories of a second order linearization of control system satisfying some second order "linearized" constraints. The present paper can be considered as the continuation and the development of [10] dealing with a general control constraint $U$, where necessary conditions for a strong local minimum were obtained for a problem with a final-state inequality constraint. Here, we have added the running state constraint of inequality type. So, the main novelty of our work is in the necessary conditions for a strong local minimum in the presence of a state constraint in combination with the general control constraint and final-state constraint. Let us also emphasize, that we do not make any hypothesis like the Mangasarian-Fromovitz or the Robinson constraint qualification (which are related to the point under the study). Moreover, we investigate the optimality conditions for an arbitrary measurable optimal control.

Our way of doing essentially differs from the other approaches, used in the theory of necessary optimality conditions, as for instance, the Ekeland's variational principle (often exploited in the proofs of the Pontryagin maximum principle, but seemingly not applicable to the derivation of the second-order conditions because of the lack of smoothness of penalty terms), the method of critical directions by Dubovitskii and Milyutin [6], the theory of higher order conditions in abstract Banach spaces [17], etc. In our approach we pass from the original control system to the convexified differential inclusion

$$
\dot{x} \in \operatorname{co} f(x, U), \quad x(0)=x_{0}
$$

and then use the apparatus of the first and second order tangents and derivatives of set-valued maps elaborated in Aubin and Frankowska [2]. It may seem that such a transition from the control system to the differential inclusion considerably coarsens the problem. But this is not so, because there is a way back which allows to obtain the necessary optimality condition in the form of non-intersection of a finite number of convex sets among which all but one are open. This way back relies on some geometric properties of derivatives of set-valued maps, found in [7] for the first order derivatives and extended in [8] to second order ones and on associated variational inclusions. Once such non-intersection condition is obtained, we use a separation theorem in order to write a dual characterization for it. Then we analyze the obtained dual criterion. As a result of analysis, we come to the maximum principle and second-order necessary optimality conditions.

We would like to discuss first the main ideas behind this variational approach and point out where the difficulties are hidden. Let $X$ be a Banach space, $K \subset X$ be an arbitrary set, $\psi: X \rightarrow \mathbb{R}$ be a $C^{2}$-function and let $\bar{x} \in K$ be a point of a local minimum of $\psi$ on $K$. If $\bar{x} \in \operatorname{Int} K$, then, by the Fermat rule, $\psi^{\prime}(\bar{x})=0$. Recall that when the Fermat rule was discovered, the derivatives were not defined yet (the infinitesimal calculus was developed by Leibniz and Newton after the Fermat death in 1665). Fermat was reasoning using tangents to the graph of a function. Tangents are also naturally present when $\bar{x}$ belongs to the boundary $\partial K$ of $K$. Denote by $T_{K}^{b}(\bar{x})$ the adjacent tangent to $K$ at $\bar{x}$, consisting of all $v \in X$ such that for every $\varepsilon>0$ there exists $r(\varepsilon) \in X$ with $\bar{x}+\varepsilon v+r(\varepsilon) \in K$ for some $|r(\varepsilon)|=o(\varepsilon)$. The first-order necessary optimality condition takes then the form of a generalized Fermat rule : $\psi^{\prime}(\bar{x}) v \geq 0$ for all $v \in T_{K}^{\mathrm{b}}(\bar{x})$. In [2] the interested reader can find calculus of tangents to sets and, in particular, the exact expression of tangent cones when $K$ is described by a finite number of inequalities and equalities under the Mangasarian-Fromovitz constraints' 
qualification assumption. This leads to the celebrated Lagrange multipliers rule of Mathematical Programming. Further, this rule is abnormal whenever this constraints' qualification is violated.

Let us have a look on the second-order necessary conditions from this perspective. If $\bar{x} \in \operatorname{Int} K$, then it is well known that $\psi^{\prime \prime}(\bar{x}) \geq 0$. When $\bar{x} \in \partial K$, it may happen that $\psi^{\prime \prime}(\bar{x})(v, v)<0$ for some $v \in T_{K}^{b}(\bar{x})$.

Indeed let $X=\mathbb{R}^{2}, K=B$ (unit ball in $\mathbb{R}^{2}$ ) and

$$
\psi\left(x_{1}, x_{2}\right)=x_{1}+1-\left(x_{1}+1\right)^{2} \quad \forall x=\left(x_{1}, x_{2}\right) \in \mathbb{R}^{2} .
$$

Then $\bar{x}=(-1,0)$ is a local minimizer of $\psi$ over $B, T_{K}^{b}(\bar{x})=\mathbb{R}_{+} \times \mathbb{R}$, while $\psi^{\prime \prime}(\bar{x})=$ $\left(a_{i j}\right)$ with $a_{11}=-2$ and all other coefficients $a_{i j}$ being equal to zero. Thus the firstorder necessary condition is verified, while for any $\left(v_{1}, v_{2}\right) \in T_{K}^{b}(\bar{x})$ with $v_{1} \neq 0$ we have $\psi^{\prime \prime}(\bar{x})(v, v)<0$.

It turns out that it is more appropriate to restrict the attention to the elements of the so called critical cone

$$
\mathcal{C}:=\left\{v \in T_{K}^{b}(\bar{x}): \psi^{\prime}(\bar{x}) v=0\right\}
$$

which, in the above example, is equal to $\{0\} \times \mathbb{R}$, and moreover $\psi^{\prime \prime}(\bar{x})(v, v)=0$ for all $v \in \mathcal{C}$. We would like to underline that even the condition $\psi^{\prime \prime}(\bar{x})(v, v) \geq 0 \forall v \in \mathcal{C}$ is not necessary for a local minimum at $\bar{x}$ (the corresponding counterexample can be constructed).

The second order variational analysis deals with second order tangents to sets: with any $v \in T_{K}^{b}(\bar{x})$ we associate a second order tangent set $T_{K}^{b(2)}(\bar{x}, v)$ consisting of all $w \in X$ such that for every $\varepsilon>0$ there exists $r(\varepsilon) \in X$ with $\bar{x}+\varepsilon v+\varepsilon^{2} w+r(\varepsilon) \in K$ for some $|r(\varepsilon)|=o\left(\varepsilon^{2}\right)$. Then, thanks to the Taylor formulae, for every $v \in \mathcal{C}$,

$$
\psi^{\prime}(\bar{x}) w+\frac{1}{2} \psi^{\prime \prime}(\bar{x})(v, v) \geq 0 \quad \forall w \in T_{K}^{b(2)}(\bar{x}, v) .
$$

This is the second-order necessary condition for a local minimum of $\psi$ on $K$ at $\bar{x}$ relatively to the critical direction $v$. The set $T_{K}^{b(2)}(\bar{x}, v)$ is closed, in general not convex and it may be empty. If it is not empty, then the above second-order optimality condition is meaningful.

Coming back to our control problem, let $X$ be the space $C\left([0,1], \mathbb{R}^{n}\right)$ of continuous functions from $[0,1]$ into $\mathbb{R}^{n}$ and let the pair $(\bar{x}, \bar{u})$ be a strong local minimizer of our control problem. Set $K=K_{1} \cap K_{2} \cap K_{3}$, where

$$
\begin{gathered}
K_{1}=\left\{x \in C\left([0,1], \mathbb{R}^{n}\right): g_{i}(x(1)) \leq 0, i=1, \ldots, k\right\}, \\
K_{2}=\left\{x \in C\left([0,1], \mathbb{R}^{n}\right): \Phi_{j}(x(t)) \leq 0, \forall t \in[0,1], j=1, \ldots, q\right\}
\end{gathered}
$$

and $K_{3}$ be the set of all absolutely continuous trajectories $x(\cdot)$ of (1.3) corresponding to some measurable controls $u:[0,1] \rightarrow U$. Then, for every critical element $y$, the second-order necessary condition becomes

$$
g_{0}^{\prime}(\bar{x}(1)) w(1)+\frac{1}{2}\left\langle g_{0}^{\prime \prime}(\bar{x}(1)) y(1), y(1)\right\rangle \geq 0 \quad \forall w \in T_{K}^{b(2)}(\bar{x}, y) .
$$

(In Section 3 we consider a particular subset of critical elements $y$, which is convenient for the purpose of this work.) 
If $g_{0}^{\prime}(\bar{x}(1))=0$, then every $y \in T_{K}^{b}(\bar{x})$ is critical and it is not difficult to realise that the second-order condition is: $\left\langle g_{0}^{\prime \prime}(\bar{x}(1)) y(1), y(1)\right\rangle \geq 0$ for every $y \in T_{K}^{b}(\bar{x})$ (even if $\left.T_{K}^{b(2)}(\bar{x}, y)=\emptyset\right)$.

Assume next that $g_{0}^{\prime}(\bar{x}(1)) \neq 0$ and fix a critical element $y$. Then the set

$$
\tilde{Q}:=\left\{z \in \mathbb{R}^{n}: g_{0}^{\prime}(\bar{x}(1)) z+\frac{1}{2}\left\langle g_{0}^{\prime \prime}(\bar{x}(1)) y(1), y(1)\right\rangle<0\right\}
$$

is nonempty, open and convex.

Even though, using [2, p. 177] and a constraint qualification assumption, it is possible to express $T_{K_{i}}^{b}(\bar{x})$ and $T_{K_{i}}^{b(2)}(\bar{x}, y)$ for $i=1,2$ and to show that they are convex, in general, we only have the relation

$$
T_{K}^{b(2)}(\bar{x}, y) \subset T_{K_{1}}^{b(2)}(\bar{x}, y) \cap T_{K_{2}}^{b(2)}(\bar{x}, y) \cap T_{K_{3}}^{b(2)}(\bar{x}, y),
$$

that may be strict. For this reason in (1.6) the set $T_{K}^{b(2)}(\bar{x}, y)$ can not be replaced by the intersection of sets $T_{K_{i}}^{b(2)}(\bar{x}, y)$.

Let us assume that we can find nonempty convex subsets $Q_{i} \subset T_{K_{i}}^{b(2)}(\bar{x}, y), i=$ $1,2,3$ such that $Q:=Q_{1} \cap Q_{2} \cap Q_{3} \subset T_{K}^{b(2)}(\bar{x}, y)$. Define the trace operator $\gamma$ on $X$ by $\gamma(x)=x(1)$ and let $\gamma^{*}: \mathbb{R}^{n} \rightarrow X$ be its adjoint. Clearly the set $\gamma(Q)$ has an empty intersection with $\tilde{Q}$. Thus, denoting by $\gamma^{-1}$ the (set-valued) inverse of $\gamma$, we deduce that $Q \cap \gamma^{-1}(\tilde{Q})=\emptyset$. Moreover $\gamma^{-1}(\tilde{Q})$ is nonempty, open and convex. If the interiors of sets $Q_{1}, Q_{2}$ are also nonempty, then, by a separation theorem (see Lemma 2.2 below), we get the following necessary optimality condition: there exist $p_{i} \in C\left([0,1], \mathbb{R}^{n}\right)^{*}, i=1,2,3$ not vanishing simultaneously such that for $p_{4}:=p_{1}+p_{2}+p_{3}$ we have

$$
\inf p_{1}\left(Q_{1}\right)+\inf p_{2}\left(Q_{2}\right)+\inf p_{3}\left(Q_{3}\right) \geq \sup p_{4}\left(\gamma^{-1}(\tilde{Q})\right) .
$$

Consider the closed convex cone $Q_{4}=\left\{z \in \mathbb{R}^{n}: g_{0}^{\prime}(\bar{x}(1)) z \leq 0\right\}$ and notice that its negative polar $Q_{4}^{-}$is equal to $\mathbb{R}_{+} g_{0}^{\prime}(\bar{x}(1))$. Since for every $v \in \tilde{Q}$ we have $\gamma^{-1}(v)+\gamma^{-1}\left(Q_{4}\right) \subset \gamma^{-1}(\tilde{Q})$, the last inequality yields

$$
\sup p_{4}\left(\gamma^{-1}\left(Q_{4}\right)\right) \leq 0
$$

The operator $\gamma$ being surjective, this and a well known result of convex analysis imply $p_{4}=\gamma^{*}\left(p_{0}\right)$ for some $p_{0} \in Q_{4}^{-}$. Let $\lambda \geq 0$ be such that $p_{0}=\lambda g_{0}^{\prime}(\bar{x}(1))$. Consequently,

$$
\inf p_{1}\left(Q_{1}\right)+\inf p_{2}\left(Q_{2}\right)+\inf p_{3}\left(Q_{3}\right) \geq \lambda \sup g_{0}^{\prime}(\bar{x}(1))(\tilde{Q})=-\frac{\lambda}{2}\left\langle g_{0}^{\prime \prime}(\bar{x}(1)) y(1), y(1)\right\rangle .
$$

This general statement has next to be made more precise by finding appropriate sets $Q_{i}$ and characterizing elements $p_{i}$ involved in the separation theorem. Observe that the family $\left\{p_{1}, p_{2}, p_{3}\right\}$ depends on the choice of the sets $Q_{i}$, and that larger are the sets $Q_{i}$, more informative are second-order necessary conditions associated to a fixed critical $y$. While investigation of second order tangents to $K_{1}$ and $K_{2}$ can be conducted by methods of functional analysis, the set $K_{3}$ is much more complex, being the set of trajectories of a nonlinear control system. To get some first-order tangents to $K_{3}$ one may consider the classical linearization of the control system at $(\bar{x}, \bar{u})$ with respect to both states and controls, where $\bar{u}$ is a control corresponding to $\bar{x}$. Then, by the variational equation, trajectories of the linearized system

$$
y^{\prime}=f_{x}(\bar{x}(t), \bar{u}(t)) y+f_{u}(\bar{x}(t), \bar{u}(t)) v(t), \quad v(t) \in T_{U}^{b}(\bar{u}(t)), \quad y(0)=0
$$


are tangent to $K_{3}$ at $\bar{x}$. This subset of tangents, however, allows to deduce only the weak form of the maximum principle, see for instance [9], where the Mayer problem was considered in the absence of state and final-point constraints.

Actually, the first order linearization of the differential inclusion $(1.5)$ at $(\bar{x}(\cdot), \dot{\bar{x}}(\cdot))$ provides a larger set of tangents to $K_{3}$ at $\bar{x}$ and has led to the strong form of the maximum principle for a differential inclusion problem, cf. [7]. In the same vein, the second order linearization was investigated in [8] and did allow to get second order tangents to $K_{3}$. This second order linearization involves second order derivatives of set-valued maps. Let us recall that first order derivatives of set-valued maps can be defined using tangent cones to their graphs. Such geometric definition of set-valued derivatives goes back to [1] and takes a direct inspiration from the Fermat approach to optimization.

We next consider a subsystem of the second order linearization having nice convexity properties and deduce the second-order necessary conditions from a separation theorem. This approach leads to a unified way to both first- and second-order necessary conditions and also brings some light on their normality, see also [4] for related first order results.

Our main goal is to derive second-order optimality conditions for strong local minimum without imposing structural assumptions on optimal control and on control constraints. In general the obtained maximum principle can be abnormal and even degenerate. We provide a sufficient condition for its normality in Remark 4.1.

The paper is organized as follows. Section 2 is devoted to some results from the convex, set-valued and functional analysis which are used in the proof of the main theorem. Our main result (Theorem 3.3) is given in Section 3 where also a subset of the critical directions is defined, while Section 4 is devoted to its proof. First, in subsection 4.1, we associate with a strong local minimizer $\bar{x}$ and a critical element $y(\cdot)$ a "second-order variational inclusion" that gives rise to some second order tangents to $K_{3}$ at $(\bar{x}, y)$ and introduce a subset of $T_{K_{3}}^{b(2)}(\bar{x}, y)$ whose closure $\mathcal{E}^{2}$ in the space $C\left([0,1], \mathbb{R}^{n}\right)$ is convex. Next, in subsection 4.2 , we define open convex sets of "second-order decrease" of the cost, of the endpoint constraints, and of the state constraints, and prove that the intersection of these sets has no common points with $\mathcal{E}^{2}$. In subsection 4.3 we apply the separation Theorem 2.1 to the system of disjoint convex sets from subsection 4.2 and analyse the separating functionals. This allows to complete the proof of the main Theorem 3.3. In Section 5, we consider two special cases for the set $U$ : when $U$ is a, not necessarily convex, polytope and when $U$ is given by inequalities with positively independent gradients of active constraints, where the main result takes a simpler form.

\section{Preliminaries.}

2.1. Some elements of convex analysis. For a Banach space $X$ we denote by $X^{*}$ its dual and by $\langle\cdot, \cdot\rangle$ the duality pairing on $X^{*} \times X$. Consider the following system in $X$ :

$$
\text { (S) }\left\{\begin{array}{l}
\left\langle l_{i}, x\right\rangle+m_{i}<0, \quad i=0, \ldots, k, \\
\varphi_{j}\left(P_{j} x+z_{j}\right)<0, \quad j=1, \ldots, q, \\
x \in M,
\end{array}\right.
$$

where $M \subset X$ is a nonempty convex set, $l_{i} \in X^{*}, m_{i} \in \mathbb{R}$ for each $i=0, \ldots, k$, while $P_{j}: X \rightarrow Z_{j}$ is a continuous linear operator mapping $X$ into another Banach space $Z_{j}, z_{j} \in Z_{j}$ is a fixed element, $\varphi_{j}: Z_{j} \rightarrow \mathbb{R}$ is a locally bounded, convex and positively homogeneous mapping for every $j=1, \ldots, q$. 
For each $j=1, \ldots, q$ denote by $\partial \varphi_{j}(0)$ the subdifferential of $\varphi_{j}$ at zero, i.e. the set $\left\{z_{j}^{*} \in Z_{j}^{*}:\left\langle z_{j}^{*}, z\right\rangle \leq \varphi_{j}(z) \quad \forall z \in Z_{j}\right\}$. Recall that it is a nonempty, convex, bounded and weakly* closed set.

THEOREM 2.1. The system $(S)$ is incompatible if and only if there exist reals $\alpha_{i} \geq 0, i=0, \ldots, k, \beta_{j} \geq 0, j=1, \ldots, q$, and linear functionals

$$
x^{*} \in X^{*}, \quad z_{j}^{*} \in \partial \varphi_{j}(0), \quad j=1, \ldots, q,
$$

such that the following three conditions hold:

$$
\begin{gathered}
\sum_{i=0}^{k} \alpha_{i}+\sum_{j=1}^{q} \beta_{j}=1, \quad \sum_{i=0}^{k} \alpha_{i} l_{i}+\sum_{j=1}^{q} \beta_{j} P_{j}^{*} z_{j}^{*}=x^{*}, \\
\sum_{i=0}^{k} \alpha_{i} m_{i}+\sum_{j=1}^{q} \beta_{j}\left\langle z_{j}^{*}, z_{j}\right\rangle+\inf \left\langle x^{*}, M\right\rangle \geq 0,
\end{gathered}
$$

where $\inf \left\langle x^{*}, M\right\rangle:=\inf _{x \in M}\left\langle x^{*}, x\right\rangle=\inf x^{*}(M)$.

Furthermore, $\alpha_{0}>0$ whenever there exists $x \in M$ such that

$$
\left\langle l_{i}, x\right\rangle+m_{i}<0, i=1, \ldots, k, \quad \varphi_{j}\left(P_{j} x+z_{j}\right)<0, j=1, \ldots, q .
$$

The last statement of the above theorem allows to get second-order optimality conditions in the normal form, see Remark 4.1 below. The proof is based on the four lemmas stated below. Two of them, Lemmas 2.3 and 2.5, first appeared in [16], in Russian. For the sake of completeness, we provide their proofs in the Appendix.

Lemma 2.2 (Dubovitskii-Milyutin [6]). Let $X$ be a Banach space, $M_{1}, \ldots, M_{k}$ be nonempty open convex sets in $X$, and $M$ be a nonempty convex set in $X$. Then

$$
M_{1} \cap \ldots \cap M_{k} \cap M=\emptyset
$$

if and only if there are $x_{1}^{*}, \ldots, x_{k}^{*}, x^{*} \in X^{*}$, not vanishing simultaneously, such that

$$
\begin{gathered}
x_{1}^{*}+\ldots+x_{k}^{*}+x^{*}=0, \\
\inf x_{1}^{*}\left(M_{1}\right)+\ldots+\inf x_{k}^{*}\left(M_{k}\right)+\inf x^{*}(M) \geq 0 .
\end{gathered}
$$

Remark 2.1. (a) In Lemma 2.2 define $M_{k+1}:=M$ and $x_{k+1}^{*}:=x^{*}$. If in addition, for some $i \in\{1, \ldots, k+1\}$, there is a nonempty cone $C_{i} \subset X$ and $x_{i} \in X$ such that $x_{i}+C_{i} \subset M_{i}$, then we also have

$$
-x_{i}^{*} \in C_{i}^{-}:=\left\{p \in X^{*}:\langle p, x\rangle \leq 0, \quad \forall x \in C_{i}\right\} .
$$

(b) Lemma 2.2 implies: if (2.2), (2.3) hold true for a nontrivial family $x_{1}^{*}, \ldots, x_{k}^{*}, x^{*} \in$ $X^{*}, k \geq 2$, and $M_{2} \cap \ldots \cap M_{k} \cap M \neq \emptyset$, then $x_{1}^{*} \neq 0$.

Proof of Lemma 2.2 We follow [20]. If (2.1) holds, then define the space $X^{k}:=$ $\underbrace{X \times X \times \ldots \times X}_{k}$ and the open convex set

$$
A:=\left\{\left(x_{1}-x, \ldots, x_{k}-x\right) \in X^{k}: x_{1} \in M_{1}, \ldots, x_{k} \in M_{k}, x \in M\right\} .
$$


Since $0 \notin A$, by the separation theorem, there exists a $0 \neq \hat{x}^{*} \in\left(X^{k}\right)^{*}$ such that $\left\langle\hat{x}^{*}, a\right\rangle \geq 0$ for every $a \in A$. Let $\hat{x}^{*}=\left(x_{1}^{*}, \ldots, x_{k}^{*}\right)$, where $x_{i}^{*} \in X^{*}, i=1, \ldots, k$. Then

$$
\left\langle x_{1}^{*}, x_{1}-x\right\rangle+\ldots+\left\langle x_{k}^{*}, x_{k}-x\right\rangle \geq 0 \text { for all } x_{1} \in M_{1}, \ldots, x_{k} \in M_{k}, x \in M .
$$

Setting $x^{*}=-\sum_{i=1}^{k} x_{i}^{*}$ yields (2.2) and (2.3).

Vice versa, let (2.2) and (2.3) hold for a nontrivial collection $x_{1}^{*}, \ldots, x_{k}^{*}, x^{*}$, but there is an element $x_{0} \in M_{1} \cap \ldots \cap M_{k} \cap M$. The non-triviality of $x_{1}^{*}, \ldots, x_{k}^{*}, x^{*}$ and (2.2) imply that the collection $x_{1}^{*}, \ldots, x_{k}^{*}$ is nontrivial. Let $x_{j}^{*} \neq 0$. Since the set $M_{j}$ is open, we have $\left\langle x_{j}^{*}, x_{0}\right\rangle>\inf \left\langle x_{j}^{*}, M_{j}\right\rangle$, and then (2.3) implies $\left\langle x_{1}^{*}, x_{0}\right\rangle+\ldots+\left\langle x_{k}^{*}, x_{0}\right\rangle+$ $\left\langle x^{*}, x_{0}\right\rangle>0$. The latter contradicts $(2.2)$ and ends the proof.

For a nonempty convex set $M \subset X$ define

$$
M^{d}=\left\{x^{*} \in X^{*}: \inf x^{*}(M)>-\infty\right\} .
$$

Let $F: X \rightarrow \mathbb{R}$ be a continuous convex function. The Fenchel conjugate of $F$ is defined by $F^{*}\left(x^{*}\right)=\sup _{x \in X}\left(\left\langle x^{*}, x\right\rangle-F(x)\right)$ for all $x^{*} \in X^{*}$. Recall that the function $F^{*}: X^{*} \rightarrow \mathbb{R} \cup\{+\infty\}$ is convex, weakly* lower semicontinuous, and its domain $\operatorname{dom} F^{*}:=\left\{x^{*} \in X^{*}: F^{*}\left(x^{*}\right)<+\infty\right\}$ is nonempty.

Lemma 2.3. Suppose that the set $M=\{x \in X: F(x)<0\}$ is nonempty. Then for every $x^{*} \in M^{d}$ there exists an $x_{1}^{*} \in \operatorname{dom} F^{*}$ and an $\alpha \geq 0$ such that $x^{*}=-\alpha x_{1}^{*}$ and $\inf x^{*}(M)=-\alpha F^{*}\left(x_{1}^{*}\right)$.

The next result follows from Lemmas 2.2 and 2.3. (it was first stated in [17] without proof.)

Lemma 2.4. Let $F_{0}(\cdot), \ldots, F_{k}(\cdot)$ be convex continuous functions on $X$, and let $M \subset X$ be a nonempty convex set. The system of conditions

$$
F_{i}(x)<0 \quad(i=0,1, \ldots, k), \quad x \in M
$$

is incompatible if and only if there is a tuple $\left(\alpha_{0}, \ldots, \alpha_{k}, x_{0}^{*}, \ldots, x_{k}^{*}, x^{*}\right)$ such that

$$
\begin{gathered}
\alpha_{i} \geq 0 \quad \forall i, \quad \sum_{i=0}^{k} \alpha_{i}=1, \\
x_{i}^{*} \in \operatorname{dom} F_{i}^{*} \quad \forall i, \quad x^{*} \in M^{d}, \\
x^{*}=\sum_{i=0}^{k} \alpha_{i} x_{i}^{*}, \\
\inf x^{*}(M) \geq \sum_{i=0}^{k} \alpha_{i} F_{i}^{*}\left(x_{i}^{*}\right) .
\end{gathered}
$$

Proof First let us assume that there is $j \in\{0, \ldots, k\}$ such that the set $\{x \in X$ : $\left.F_{j}(x)<0\right\}$ is empty. It means that $F_{j}(x) \geq 0$ for all $x \in X$. Set $x_{j}^{*}=0$. Then obviously $x_{j}^{*} \in \operatorname{dom} F_{j}^{*}$ and $F_{j}^{*}\left(x_{j}^{*}\right) \leq 0$. Take $\alpha_{j}=1, x^{*}=0$, and set all other $\alpha_{i}=0$, while choosing $x_{i}^{*} \in \operatorname{dom} F_{i}^{*}$ arbitrary (for all $i \neq j$ ). Then all conditions 
(2.4)-(2.7) are fulfilled. Next, if $\left\{x \in X: F_{i}(x)<0\right\} \neq \emptyset$ for all $i=0, \ldots, k$, then apply Lemmas 2.2 and 2.3 .

Lemma 2.5. Let $X, Y$ be Banach spaces, $\varphi: Y \rightarrow \mathbb{R}$ be a continuous convex function on $Y, A: X \rightarrow Y$ be a continuous linear operator and $y_{0} \in Y$. Then the function $F(x):=\varphi\left(y_{0}+A x\right)$ is continuous and convex on $X$, and for any $x^{*} \in \operatorname{dom} F^{*}$ there exists $y^{*} \in \operatorname{dom} \varphi^{*}$ such that $x^{*}=A^{*} y^{*}$ and $F^{*}\left(x^{*}\right)=\varphi^{*}\left(y^{*}\right)-\left\langle y^{*}, y_{0}\right\rangle$.

Proof of Theorem 2.1 Note that if $\varphi: Y \rightarrow \mathbb{R}$ is continuous, convex and positively homogeneous, then $\operatorname{dom} \varphi^{*}=\partial \varphi(0), \varphi^{*}\left(y^{*}\right)=0$ for every $y^{*} \in \partial \varphi(0)$ and hence in Lemma 2.5 we have: $F^{*}\left(x^{*}\right)=-\left\langle y^{*}, y_{0}\right\rangle$. Therefore, Lemmas 2.4 and 2.5 imply the first statement of Theorem 2.1. The last one, follows from Remark 2.1 (b).

We will also need the following result. In the space $C([0,1])$ of real valued continuous functions, consider a convex and positively homogeneous function

$$
\varphi(z)=\max _{t \in \mathcal{M}} z(t) \quad \forall z \in C([0,1]),
$$

where $\mathcal{M} \subset[0,1]$ is a given closed set.

Lemma 2.6 ([6]). The sub-differential $\partial \varphi(0)$ of $\varphi$ defined by (2.8) consists of all probability measures $\mu$ with $\operatorname{supp} \mu \subset \mathcal{M}$.

Proof We provide here a simpler proof than in [6]. Again we follow [20]. Let $\mu \in \partial \varphi(0)$. Since $[0,1]$ is compact, $\mu$ is a Borel measure. Obviously,

$$
\int_{[0,1]} z(t) \mathrm{d} \mu \leq \max _{t \in \mathcal{M}} z(t), \quad \forall z \in C([0,1]) .
$$

Take any $z \in C([0,1])$ vanishing on $\mathcal{M}$. Then it follows from (2.9) that $\int_{[0,1]} z(t) \mathrm{d} \mu \leq$ 0 and similarly $\int_{[0,1]}(-z(t)) \mathrm{d} \mu \leq 0$, whence $\int_{[0,1]} z(t) \mathrm{d} \mu=0$. This means that $\operatorname{supp} \mu \subset \mathcal{M}$. Let $z(\cdot) \geq 0$. Then again it follows from (2.9) that $\int_{[0,1]}(-z(t)) \mathrm{d} \mu \leq 0$, and hence $\int_{[0,1]} z(t) \mathrm{d} \mu \geq 0$ for all $z(\cdot) \geq 0$. This implies that $\mu \geq 0$. Now taking $z(t) \equiv 1$ and $z(t) \equiv-1$, we get from $(2.9): \int_{[0,1]} \mathrm{d} \mu \leq 1$ and $\int_{[0,1]}(-1) \mathrm{d} \mu \leq-1$, respectively, whence $\int_{[0,1]} \mathrm{d} \mu=1$.

Vice versa, let $\mu$ be a probability measure, concentrated on $\mathcal{M}$. Take any $z(\cdot) \in$ $C([0,1])$ and set $c:=\max _{\mathcal{M}} z(t)$. Then $z(t)-c \leq 0$ on $\mathcal{M}$, and thus $\int_{[0,1]}(z(t)-c) \mathrm{d} \mu \leq$ 0 since $\mu$ is nonnegative and concentrated on $\mathcal{M}$. This implies that

$$
\int_{[0,1]} z(t) \mathrm{d} \mu \leq \int_{[0,1]} c \mathrm{~d} \mu=c=\max _{\mathcal{M}} z(t)=\varphi(z),
$$

since $\int_{[0,1]} \mathrm{d} \mu=1$. Hence $\mu \in \partial \varphi(0)$ and the lemma is proved.

2.2. Some elements of set-valued analysis. Let $X$ be a Banach space and $\emptyset \neq K \subset X$. The distance from $x \in X$ to $K$ is defined by $\mathrm{d}_{K}(x)=\inf _{x^{\prime} \in K}\left|x^{\prime}-x\right|$. For a family $\left\{A_{h}\right\}_{h>0}$ of subsets of $X$ the lower set limit is defined by

$$
v \in \operatorname{Liminf}_{h \rightarrow 0+} A_{h} \Leftrightarrow \lim _{h \rightarrow 0+} \mathrm{d}_{A_{h}}(v)=0 .
$$

The adjacent tangent cone to $K$ at $\bar{x} \in K$ is the closed cone

$$
T_{K}^{b}(\bar{x})=\operatorname{Liminf}_{h \rightarrow 0+} \frac{K-\bar{x}}{h}
$$


and the second-order adjacent set to $K$ at $(\bar{x}, v) \in K \times X$ is the closed set

$$
T_{K}^{b(2)}(\bar{x}, v)=\operatorname{Liminf}_{h \rightarrow 0+} \frac{K-\bar{x}-h v}{h^{2}} .
$$

When $K$ is convex, to be in line with the usual notations of convex analysis, we will also write $T_{K}(\bar{x})$ instead of $T_{K}^{b}(\bar{x})$.

A set-valued map $F: \mathbb{R}^{n} \rightsquigarrow \mathbb{R}^{m}$ is called locally Lipschitz if for every $R>0$ there exists $c_{R} \geq 0$ such that $F(x) \subset F(y)+c_{R}|x-y| B_{m}$ for all $x, y \in B(0, R)$, where $B_{m}$ denotes the closed unit ball in $\mathbb{R}^{m}$.

Consider a locally Lipschitz set-valued map $F: \mathbb{R}^{n} \rightsquigarrow \mathbb{R}^{m}$. Recall that the adjacent derivative of $F$ at a point $(x, y) \in \mathbb{R}^{n} \times \mathbb{R}^{m}$ with $y \in F(x)$, in the direction $u \in \mathbb{R}^{n}$, is defined by

$$
\mathrm{d} F(x, y)(u)=\operatorname{Liminf}_{h \rightarrow 0+} \frac{F(x+h u)-y}{h} .
$$

For $u_{1} \in \mathbb{R}^{n}$ and $v_{1} \in \mathrm{d} F(x, y)\left(u_{1}\right)$, the second-order adjacent derivative of $F$ at $\left(x, y, u_{1}, v_{1}\right)$ in the direction $u_{2} \in \mathbb{R}^{n}$ is defined by

$$
\mathrm{d}^{2} F\left(x, y, u_{1}, v_{1}\right)\left(u_{2}\right)=\operatorname{Liminf}_{h \rightarrow 0+} \frac{F\left(x+h u_{1}+h^{2} u_{2}\right)-y-h v_{1}}{h^{2}} .
$$

If, in addition, $F(\cdot)$ has convex values, then the following is true, cf. [7]: for any $u \in \mathbb{R}^{m}$ the set $\mathrm{d} F(x, y)(u)$ is convex and

$$
\mathrm{d} F(x, y)(0)=T_{F(x)}(y), \quad \mathrm{d} F(x, y)(u)+T_{F(x)}(y)=\mathrm{d} F(x, y)(u) .
$$

Moreover, by [8, Proposition 2.11], for all $v_{1} \in d F(x, y)\left(u_{1}\right)$ and every $u_{2} \in \mathbb{R}^{n}$,

$$
\mathrm{d}^{2} F\left(x, y, u_{1}, v_{1}\right)\left(u_{2}\right)+T_{F(x)}(y)=\mathrm{d}^{2} F\left(x, y, u_{1}, v_{1}\right)\left(u_{2}\right) .
$$

In the above, by convention, $\emptyset+A=\emptyset$ for any set $A \subset \mathbb{R}^{m}$.

3. Main result. Denote by $W^{1,1}\left([0,1], \mathbb{R}^{n}\right)$ the Sobolev space of absolutely continuous functions $x:[0,1] \rightarrow \mathbb{R}^{n}$ with the norm $\|x(\cdot)\|_{1,1}=|x(0)|+\int_{0}^{1}|\dot{x}(t)| \mathrm{d} t$ and by $L^{\infty}\left([0,1], \mathbb{R}^{m}\right)$ the space of measurable essentially bounded functions $u:[0,1] \rightarrow$ $\mathbb{R}^{m}$ with the norm $\|u(\cdot)\|_{\infty}=\operatorname{ess}_{\sup _{[0,1]}}|u(t)|$, where $|\cdot|$ denotes the Euclidean norm. Define the space

$$
\Xi:=W^{1,1}\left([0,1], \mathbb{R}^{n}\right) \times L^{\infty}\left([0,1], \mathbb{R}^{m}\right)
$$

with the norm of $\xi(\cdot)=(x(\cdot), u(\cdot)) \in \Xi$ given by $\|\xi(\cdot)\|=\|x(\cdot)\|_{1,1}+\|u(\cdot)\|_{\infty}$.

Any trajectory-control pair $(x, u) \in \Xi$ satisfying (1.2)-(1.4) is called admissible. Recall that a weak local minimum is a local minimum over admissible pairs in the space $\Xi$. Further, an admissible $(\bar{x}, \bar{u})$ is called a strong local minimizer if there exists an $\varepsilon>0$ such that $J(x, u) \geq J(\bar{x}, \bar{u})$ for any admissible $(x, u) \in \Xi$ such that $\|x-\bar{x}\|_{\infty}<\varepsilon$. Obviously, under our assumptions, any strong local minimizer is a weak local minimizer. 
3.1. First order necessary conditions. The Hamiltonian $\mathcal{H}: \mathbb{R}^{n} \times \mathbb{R}^{m} \times$ $\mathbb{R}^{n} \rightarrow \mathbb{R}$ and the terminal Lagrange function $l: \mathbb{R}^{n} \times \mathbb{R}^{k} \rightarrow \mathbb{R}$ are defined by

$$
\mathcal{H}(x, u, p)=p f(x, u), \quad l(x, \alpha)=\sum_{i=0}^{k} \alpha_{i} g_{i}(x),
$$

where $p=\left(p_{1}, \ldots, p_{n}\right)$ is considered as a row vector and $\alpha=\left(\alpha_{0}, \ldots, \alpha_{k}\right)$. Denote by

$$
\begin{aligned}
& K_{i}^{f}=\left\{x \in \mathbb{R}^{n}: g_{i}(x) \leq 0\right\}, \quad i=1, \ldots, k, \\
& K_{j}=\left\{x \in \mathbb{R}^{n}: \Phi_{j}(x) \leq 0\right\}, \quad j=1, \ldots, q .
\end{aligned}
$$

Then for every $x \in \partial K_{i}^{f}$ we have $g_{i}(x)=0$ and similarly for $\Phi_{j}$.

Let $(\bar{x}, \bar{u})$ be a strong local minimizer in problem (1.1)-(1.4). The first-order necessary conditions for a strong local minimum are as follows: there exist

$$
\alpha=\left(\alpha_{0}, \ldots, \alpha_{k}\right), \quad \alpha \geq 0 \quad \text { such that } \alpha_{i}=0 \text { if } \bar{x}(1) \in \operatorname{Int} K_{i}^{f}, \quad i=1, \ldots, k,
$$

positive Borel measures $\mu_{j}$ on $[0,1]$ with

$$
\operatorname{supp} \mu_{j} \subset\left\{t \in[0,1]: \bar{x}(t) \in \partial K_{j}\right\}, \quad j=1, \ldots, q,
$$

and a right-continuous function of bounded variation $p:[0,1] \rightarrow \mathbb{R}^{n}$ such that

$$
\begin{gathered}
-\mathrm{d} p(t)=\mathcal{H}_{x}(\bar{x}(t), \bar{u}(t), p(t)) \mathrm{d} t+\sum_{j=1}^{q} \Phi_{j}^{\prime}(\bar{x}(t)) \mathrm{d} \mu_{j}(t), \\
p(1)=l_{x}(\bar{x}(1), \alpha), \\
\min _{v \in U} \mathcal{H}(\bar{x}(t), v, p(t))=\mathcal{H}(\bar{x}(t), \bar{u}(t), p(t)) \quad \text { a.e. in } \quad[0,1], \\
\sum_{i=0}^{k} \alpha_{i}+\sum_{j=1}^{q} \mu_{j}([0,1])=1 .
\end{gathered}
$$

In (3.3) the equality has to be understood in the sense of measures, and a.e. in (3.5) refers to the Lebesgue measure.

For $\mu=\left(\mu_{1}, \ldots, \mu_{q}\right)$, denote by $M(\bar{x}, \bar{u})$ the set of all triples $(\alpha, p, \mu)$, satisfying the minimum principle conditions (3.1)-(3.6). The following result is well known.

THEOREM 3.1. If $(\bar{x}, \bar{u})$ is a strong local minimizer, then $M(\bar{x}, \bar{u}) \neq \emptyset$.

Let $(\alpha, p, \mu) \in M(\bar{x}, \bar{u})$. Then the minimum condition (3.5) implies

$$
\mathcal{H}_{u}[t] v \geq 0 \quad \forall v \in T_{U}^{b}(\bar{u}(t)) \text { for a.e. } t \in[0,1],
$$

where $[t]:=(\bar{x}(t), \bar{u}(t), p(t))$. (In what follows we will often use this abbreviation for, possibly different, sets of time dependent functions.) Denote by $\Lambda(\bar{x}, \bar{u})$ the set of all $(\alpha, p, \mu)$ satisfying (3.1)-(3.4) and (3.6), (3.7). Clearly, $M(\bar{x}, \bar{u}) \subset \Lambda(\bar{x}, \bar{u})$. The condition $\Lambda(\bar{x}, \bar{u}) \neq \emptyset$ is called the "weak local minimum principle". (It is the firstorder necessary optimality condition for a weak local minimum in our problem.) 


\subsection{Second-order conditions.}

Critical cone. Denote by $\mathcal{C}(\bar{x}, \bar{u})$ the set of all pairs $(y(\cdot), u(\cdot)) \in \Xi$ such that

$$
\begin{gathered}
g_{i}^{\prime}(\bar{x}(1)) y(1) \leq 0, \quad i \in I_{g} \cup\{0\}, \\
\Phi_{j}^{\prime}(\bar{x}(t)) y(t) \leq 0 \quad \forall t \in \mathcal{M}_{j 0}, \quad j=1, \ldots, q, \\
\dot{y}(t)=f_{x}[t] y(t)+f_{u}[t] u(t) \quad \text { a.e. on }[0,1], \quad y(0)=0, \\
u(t) \in T_{U}^{b}(\bar{u}(t)) \quad \text { a.e. in }[0,1],
\end{gathered}
$$

where $[t]:=(\bar{x}(t), \bar{u}(t))$,

$I_{g}=\left\{i \in\{1, \ldots, k\}: \bar{x}(1) \in \partial K_{i}^{f}\right\}, \quad \mathcal{M}_{j 0}=\left\{t \in[0,1]: \bar{x}(t) \in \partial K_{j}\right\}, \quad j=1, \ldots, q$.

$\mathcal{C}(\bar{x}, \bar{u})$ is called in this paper the critical cone (it is possible to define larger critical sets that we do not do here). The elements of the critical cone $\mathcal{C}(\bar{x}, \bar{u})$ have the following important properties.

Lemma 3.2. Let $(\alpha, p, \mu) \in \Lambda(\bar{x}, \bar{u})$. Then for any $(y, u) \in \mathcal{C}(\bar{x}, \bar{u})$,

$$
\begin{gathered}
\alpha_{i} g_{i}^{\prime}(\bar{x}(1)) y(1)=0, \quad i=0, \ldots, k ; \quad \mathcal{H}_{u}[t] u(t)=0 \quad \text { a.e. in }[0,1], \\
\Phi_{j}^{\prime}(\bar{x}(t)) y(t)=0 \quad \mu_{j} \text {-a.e. in }[0,1], \quad j=1, \ldots, q .
\end{gathered}
$$

Proof By (3.3), (3.4) (3.10) and the condition $y(0)=0$, we get

$$
\begin{aligned}
\int_{0}^{1} \mathcal{H}_{u}[t] u(t) \mathrm{d} t & -\sum_{j=1}^{q} \int_{[0,1]} \Phi_{j}^{\prime}(\bar{x}(t)) y(t) \mathrm{d} \mu_{j}=p(1) y(1) \\
& =l_{x}(\bar{x}(1), \alpha) y(1)=\sum_{i=0}^{k} \alpha_{i} g_{i}^{\prime}(\bar{x}(1)) y(1) .
\end{aligned}
$$

Consequently,

$$
\sum_{i=0}^{k} \alpha_{i} g_{i}^{\prime}(\bar{x}(1)) y(1)-\int_{0}^{1} \mathcal{H}_{u}[t] u(t) \mathrm{d} t+\sum_{j=1}^{q} \int_{[0,1]} \Phi_{j}^{\prime}(\bar{x}(t)) y(t) \mathrm{d} \mu_{j}=0 .
$$

Since by (3.7), (3.8), and (3.9) all summand in the left-hand side of this equality are nonpositive, it follows that all of them are equal to zero. Moreover, the conditions $\int_{0}^{1} \mathcal{H}_{u}[t] u(t) \mathrm{d} t=0$ and $\mathcal{H}_{u}[t] u(t) \geq 0$ a.e. in $[0,1]$ imply $\mathcal{H}_{u}[t] u(t)=0$ a.e. in $[0,1]$. Similarly, the conditions $\int_{0}^{1} \Phi_{j}^{\prime}(\bar{x}(t)) y(t) \mathrm{d} \mu_{j}=0, \Phi_{j}^{\prime}(\bar{x}(t)) y(t) \leq 0$ for all $t \in \mathcal{M}_{j 0}$ and (3.2) imply $\Phi_{j}^{\prime}(\bar{x}(t)) y(t)=0 \mu_{j}$ - a.e. in $[0,1], j=1, \ldots, q$.

Consequently, if, for some index $i \in\{0, \ldots, k\}$, there exists $(\alpha, p, \mu) \in \Lambda(\bar{x}, \bar{u})$ such that $\alpha_{i}>0$, then the inequality $g_{i}^{\prime}(\bar{x}(1)) y(1) \leq 0$ (in the definition of the critical cone) can be replaced by the equality $g_{i}^{\prime}(\bar{x}(1)) y(1)=0$ without affecting $\mathcal{C}(\bar{x}, \bar{u})$. Similarly for any $(\alpha, p, \mu) \in \Lambda(\bar{x}, \bar{u})$ the conditions $\mathcal{H}_{u}[t] u(t)=0$ a.e. in $[0,1]$ and $\Phi_{j}^{\prime}(\bar{x}(t)) y(t)=0 \mu_{j}$ - a.e. in $[0,1], j=1, \ldots, q$, can be added to the definition of $\mathcal{C}(\bar{x}, \bar{u})$ without affecting $\mathcal{C}(\bar{x}, \bar{u})$. 
We will also use a smaller cone of critical directions. First consider the following condition for a function $u(\cdot) \in L^{\infty}\left([0,1], \mathbb{R}^{n}\right)$ :

$$
\left\{\begin{array}{l}
\exists h_{0}>0, \quad \exists c(\cdot) \in L^{1}\left([0,1], \mathbb{R}_{+}\right) \quad \text { such that } \forall h \in\left[0, h_{0}\right] \\
\mathrm{d}_{U}(\bar{u}(t)+h u(t)) \leq c(t) h^{2} \quad \text { for a.e. } t \in[0,1] .
\end{array}\right.
$$

Note that this condition yields (3.11). Denote by $\mathcal{C}_{U}(\bar{x}, \bar{u})$ the set of all elements $(y(\cdot), u(\cdot)) \in \mathcal{C}(\bar{x}, \bar{u})$ such that $u(\cdot)$ satisfies $(3.12)$ and by $\mathcal{C}_{\Phi}(\bar{x}, \bar{u})$ the set of all elements $(y(\cdot), u(\cdot)) \in \mathcal{C}(\bar{x}, \bar{u})$ such that

$$
\exists \delta>0 \quad \text { satisfying } \max _{t \in \mathcal{M}_{j \delta}} \Phi_{j}^{\prime}(\bar{x}(t)) y(t) \leq 0, \quad \forall j=1, \ldots, q,
$$

where

$$
\mathcal{M}_{j \delta}=\left\{t \in[0,1]: \Phi_{j}(\bar{x}(t)) \geq-\delta, \mathrm{d}_{\partial K_{j}}(\bar{x}(t)) \leq \delta\right\} .
$$

Remark 3.1. If for some $j, \Phi_{j}^{\prime}(\bar{x}(t)) y(t)<0$ for all $t \in \mathcal{M}_{j 0}$, then, obviously, there exists $\delta>0$ such that $\Phi_{j}^{\prime}(\bar{x}(t)) y(t)<0$ for all $t \in \mathcal{M}_{j \delta}$.

Finally, define

$$
\mathcal{C}_{0}(\bar{x}, \bar{u}):=\mathcal{C}_{U}(\bar{x}, \bar{u}) \cap \mathcal{C}_{\Phi}(\bar{x}, \bar{u}) .
$$

We show next that a second-order necessary optimality condition, can be associated with every element of the cone $\mathcal{C}_{0}(\bar{x}, \bar{u})$.

Main theorem. For any $u:[0,1] \rightarrow \mathbb{R}^{m}$ define

$$
\begin{aligned}
V^{2}(\bar{u}, u):= & \left\{v:[0,1] \rightarrow \mathbb{R}^{m}: v(\cdot)\right. \text { is measurable, } \\
& \left.f_{u}[\cdot] v(\cdot) \text { is integrable and } v(t) \in T_{U}^{b(2)}(\bar{u}(t), u(t)) \text { a.e. }\right\},
\end{aligned}
$$

and for any $(\alpha, p, \mu) \in M(\bar{x}, \bar{u})$ and $t \in[0,1]$, set

$$
\Upsilon(u(t), p(t)):=\inf \left\{\mathcal{H}_{u}[t] v: v \in T_{U}^{b(2)}(\bar{u}(t), u(t))\right\} \in[-\infty,+\infty],
$$

where, by convention, $\inf _{\emptyset}=+\infty$. Observe that if $T_{U}^{b(2)}(\bar{u}(t), u(t))=\emptyset$ on a subset of $[0,1]$ of positive measure, then $V^{2}(\bar{u}, u)=\emptyset$.

Further, for any $(\alpha, p, \mu) \in M(\bar{x}, \bar{u})$ and $\xi=(y, u) \in \Xi$, set

$$
\begin{aligned}
\Omega(\xi, \alpha, p, \mu):= & \frac{1}{2}\left\langle l_{x x}(\bar{x}(1), \alpha) y(1), y(1)\right\rangle+\frac{1}{2} \int_{0}^{1}\left\langle\mathcal{H}^{\prime \prime}[t] \xi(t), \xi(t)\right\rangle \mathrm{d} t \\
& +\frac{1}{2} \sum_{j=1}^{q} \int_{[0,1]}\left\langle\Phi_{j}^{\prime \prime}(\bar{x}(t)) y(t), y(t)\right\rangle \mathrm{d} \mu_{j},
\end{aligned}
$$

where $\mathcal{H}^{\prime \prime}[t]$ is the Hessian of $\mathcal{H}(\cdot, \cdot, p(t))$ at $(\bar{x}(t), \bar{u}(t))$. Note that the functional $\Omega(\xi, \alpha, p, \mu)$ is quadratic in $\xi$.

Theorem 3.3 below is the main result of this paper.

THEOREM 3.3. If $(\bar{x}, \bar{u})$ is a strong local minimizer, then, for any $\xi=(y, u) \in$ $\mathcal{C}_{0}(\bar{x}, \bar{u})$ with $V^{2}(\bar{u}, u) \neq \emptyset$, there exists $(\alpha, p, \mu) \in M(\bar{x}, \bar{u})$, for which the function $\Upsilon(u(\cdot), p(\cdot))$ is integrable and the following inequality holds true:

$$
\Omega(\xi, \alpha, p, \mu)+\int_{0}^{1} \Upsilon(u(t), p(t)) \mathrm{d} t \geq 0 .
$$


Remark 3.2. Note that $(\alpha, p, \mu)$ in the above theorem depends on $\xi$ and it can be stated also in the following way: If $(\bar{x}, \bar{u})$ is a strong local minimizer, then,

$$
\inf _{\substack{\xi(y, u) \in \mathcal{C}_{0}(\bar{x}, \bar{u}) \\ V^{2}(\bar{u}, u) \neq \emptyset}} \sup _{(\alpha, p, \mu) \in M(\bar{x}, \bar{u})}\left(\Omega(\xi, \alpha, p, \mu)+\int_{0}^{1} \Upsilon(u(t), p(t)) \mathrm{d} t\right) \geq 0
$$

with the convention that the supremum over an empty set is equal to $-\infty$.

Remark 3.3. In [10], we provided examples, where, for every critical element $(x, u)$, the condition $V^{2}(\bar{u}, u) \neq \emptyset$ is fulfilled and the term $\Upsilon(u(t), p(t))$ can be computed explicitly. This is so, e.g., if $U$ is a convex polytope (then we always have $\Upsilon(u(t), p(t))=0)$, or if $U$ is given by the system of inequalities $\varphi_{i}(u) \leq 0, i=1, \ldots, r$ with $C^{2}$-functions $\varphi_{i}: \mathbb{R}^{m} \rightarrow \mathbb{R}$, having positively independent gradients of active constraints. This last case will be analyzed also in Section 5.2.

\section{Proof of Theorem 3.3.}

4.1. Set of admissible second-order variations. We first discuss the second order variations of $\bar{x}$. For every $x \in \mathbb{R}^{n}$, define the convex compact set

$$
F(x):=\operatorname{co} f(x, U) .
$$

Then, the set-valued map $F$ is locally Lipschitz continuous and locally bounded.

Let $(\bar{x}, \bar{u}) \in \Xi$ solve (1.3). Then $\bar{x}$ satisfies the differential inclusion

$$
\dot{x}(t) \in F(x(t)), \quad \text { for a.e. } t \in[0,1], \quad x(0)=x_{0} .
$$

We have shown in [10] that for a.e. $t \in[0,1]$ and any $y \in \mathbb{R}^{n}, u \in T_{U}^{b}(\bar{u}(t)), v \in$ $T_{U}^{b(2)}(\bar{u}(t), u), w \in \mathbb{R}^{n}$,

$$
\begin{gathered}
z:=f_{x}[t] y+f_{u}[t] u \in \mathrm{d} F(\bar{x}(t), \dot{\bar{x}}(t))(y), \\
f_{x}[t] w+f_{u}[t] v+\frac{1}{2} f^{\prime \prime}[t]((y, u),(y, u))+T_{F(\bar{x}(t))}(\dot{\bar{x}}(t)) \subset \mathrm{d}^{2} F(\bar{x}(t), \dot{\bar{x}}(t), y, z)(w) .
\end{gathered}
$$

The set of all absolutely continuous functions $y:[0,1] \rightarrow \mathbb{R}^{n}$ satisfying

(i) $\dot{y}(t) \in \mathrm{d} F(\bar{x}(t), \dot{\bar{x}}(t))(y(t))$ for a.e. $t \in[0,1], \quad y(0)=0$,

(ii) $\exists \alpha \in L^{1}\left([0,1], \mathbb{R}_{+}\right), \exists h_{0}>0$ such that for all $h \in\left[0, h_{0}\right]$ and a.e. $t \in[0,1]$,

$$
\operatorname{dist}_{F(\bar{x}(t)+h y(t))}(\dot{\bar{x}}(t)+h \dot{y}(t)) \leq \alpha(t) h^{2}
$$

is called the set of admissible first order variations at $\bar{x}$ and is denoted by $\mathcal{V}^{(1)}(\bar{x})$.

Lemma 4.1 ([10]). Let a pair $(y, u) \in \Xi$ solve the system

$$
\dot{y}(t)=f_{x}[t] y(t)+f_{u}[t] u(t), \quad y(0)=0,
$$

and $u$ satisfies (3.12). Then $y \in \mathcal{V}^{(1)}(\bar{x})$.

In particular, if $(y, u) \in \mathcal{C}_{0}(\bar{x}, \bar{u})$, then $y \in \mathcal{V}^{(1)}(\bar{x})$.

For a fixed $y \in \mathcal{V}^{(1)}(\bar{x})$, we abbreviate $(\bar{x}(t), \dot{\bar{x}}(t), y(t), \dot{y}(t))$ by $[t]$ and consider the set $\mathcal{V}^{(2)}(\bar{x}, y)$ of all absolutely continuous functions $w:[0,1] \rightarrow \mathbb{R}^{n}$ satisfying

$$
\dot{w}(t) \in \mathrm{d}^{2} F[t](w(t)) \text { for a.e. } t \in[0,1], \quad w(0)=0 .
$$


Let $\xi=(y, u)$ be as in Lemma 4.1 and assume that the set $V^{2}(\bar{u}, u)$ (defined by $(3.15))$ is nonempty. Consider the closed convex cone

$$
K:=\left\{\eta \in L^{1}\left([0,1], \mathbb{R}^{n}\right): \eta(t) \in T_{F(\bar{x}(t))}(\dot{\bar{x}}(t)) \text { a.e. in }[0,1]\right\}
$$

and the control system

$$
\dot{w}(t)=f_{x}[t] w(t)+f_{u}[t] v(t)+\frac{1}{2} f^{\prime \prime}[t](\xi(t), \xi(t))+\eta(t), \quad w(0)=0,
$$

where controls $\eta \in K$ and $v \in V^{2}(\bar{u}, u)$. We introduce next the set of admissible second order variations

$\tilde{\mathcal{V}}^{(2)}(\bar{x}, y)=\left\{w \in W^{1,1}\left([0,1], \mathbb{R}^{n}\right): w\right.$ solves $(4.3)$ for some $\left.\eta \in K, v \in V^{2}(\bar{u}, u)\right\}$.

Lemma $4.2([10])$. Let $(y, u)$ be as in Lemma 4.1 and $V^{2}(\bar{u}, u) \neq \emptyset$. Then

$$
\emptyset \neq \tilde{\mathcal{V}}^{(2)}(\bar{x}, y) \subset \mathcal{V}^{(2)}(\bar{x}, y)
$$

Fix any $\xi=(y, u) \in \mathcal{C}_{0}(\bar{x}, \bar{u})$ such that $V^{2}(\bar{u}, u) \neq \emptyset$.

Denote by $\imath: W^{1,1}\left([0,1], \mathbb{R}^{n}\right) \rightarrow C\left([0,1], \mathbb{R}^{n}\right)$ the natural embedding and define

$$
\mathcal{E}^{2}=\operatorname{cl}\left\{\imath\left(\tilde{\mathcal{V}}^{(2)}(\bar{x}, y)\right)\right\},
$$

where cl stands for the closure in the space $\left.C([0,1]), \mathbb{R}^{n}\right)$.

LEMMA 4.3. The closed nonempty set $\mathcal{E}^{2}$ is convex.

Proof We have to prove that $\mathcal{E}^{2}=\operatorname{co} \mathcal{E}^{2}$. The inclusion $\subset$ is obvious. To prove the opposite inclusion it is enough to show that $\mathcal{E}^{2} \supset \operatorname{co}\left\{\imath\left(\tilde{\mathcal{V}}^{(2)}(\bar{x}, y)\right)\right\}$. For this aim consider any integer $s \geq 1$, reals $\lambda_{i} \geq 0$ such that $\sum_{i=1}^{s} \lambda_{i}=1$ and $w_{i} \in \tilde{\mathcal{V}}^{(2)}(\bar{x}, y)$ for $i=1, \ldots, s$. We verify that

$$
\bar{w}:=\sum_{i=1}^{s} \lambda_{i} w_{i} \in \mathcal{E}^{2}
$$

Let $v_{i} \in V^{2}(\bar{u}, u), \eta_{i} \in K$ be controls corresponding to $w_{i}$. Then $\bar{\eta}:=\sum_{i=1}^{s} \lambda_{i} \eta_{i} \in$ $K$, because $K$ is convex. Define the integrable function

$$
\gamma(t)=\max _{1 \leq i \leq s}\left|f_{u}[t] v_{i}(t)\right|
$$

and the measurable set-valued map

$$
\Pi(t)=\left\{f_{u}[t] v_{i}(t): i=1, \ldots, s\right\} \quad \forall t \in[0,1] .
$$

Clearly $\Pi(t) \subset \gamma(t) B$ a.e. (where $B$ denotes the unit ball in $\mathbb{R}^{m}$ ) and thus $\Pi$ is integrably bounded. Furthermore, $\bar{w}$ is a trajectory of the affine with respect to controls $\pi$ system

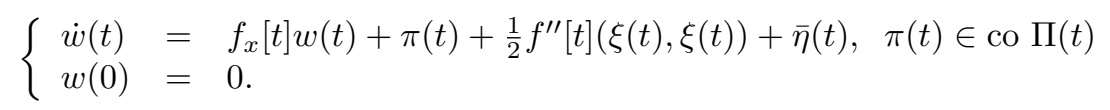


By the relaxation theorem there exists a sequence of solutions $\bar{w}^{j}$ to the control system

$$
\left\{\begin{array}{l}
\dot{w}(t)=f_{x}[t] w(t)+\pi(t)+\frac{1}{2} f^{\prime \prime}[t](\xi(t), \xi(t))+\bar{\eta}(t), \quad \pi(t) \in \Pi(t) \\
w(0)=0
\end{array}\right.
$$

converging uniformly to $\bar{w}$. For every $j$, let $\pi^{j} \in L^{1}\left([0,1], \mathbb{R}^{n}\right)$ be a control corresponding to $\bar{w}^{j}$. Since $\Pi(t)=f_{u}[t]\left\{v_{i}(t): i=1, \ldots, s\right\}$, by the measurable selection theorem, for every $j$ there exists a measurable selection $\bar{v}^{j}(t) \in\left\{v_{i}(t): i=1, \ldots, s\right\}$ a.e. such that $\pi^{j}(t)=f_{u}[t] \bar{v}^{j}(t)$ a.e. Hence $\bar{v}^{j} \in V^{2}(\bar{u}, u)$ and therefore $\bar{w}^{j} \in \tilde{\mathcal{V}}^{(2)}(\bar{x}, y)$ for every $j$, implying that $\bar{w} \in \mathcal{E}^{2}$.

Finally, we recall one more important fact needed in the proof of the main theorem. Denote by $S$ the set of all $x \in W^{1,1}\left([0,1], \mathbb{R}^{n}\right)$ such that there exists $u \in L^{\infty}([0,1], U)$ with $(x, u)$ satisfying (1.3). Recall that, by a measurable selection theorem, see for instance [2, Theorem 8.2.10], the set $S$ coincides with the set of trajectories of the differential inclusion

$$
\dot{x}(t) \in f(x(t), U) \quad \text { a.e. in } \quad[0,1], \quad x(0)=x_{0} .
$$

Thus, by [8, Theorem 3.3], for every $y \in \mathcal{V}^{(1)}(\bar{x}), w \in \mathcal{V}^{(2)}(\bar{x}, y)$ and for any sequence $h_{s} \rightarrow 0+$, there exists a sequence $x_{s} \in S, s=1, \ldots$, such that

$$
\frac{x_{s}-\bar{x}-h_{s} y}{h_{s}^{2}} \rightarrow w \quad \text { uniformly on }[0,1] \text { as } s \rightarrow \infty .
$$

We would like to underline that in [8] $F$ is supposed to satisfy also a sublinear growth assumption. However, the same proof as in [8] applies whenever $U$ is compact and $f \in C^{2}$ and so this last assumption may be skipped. It follows that

$$
x_{s}=\bar{x}+h_{s} y+h_{s}^{2} w+h_{s}^{2} r_{s}, \quad \text { where }\left\|r_{s}\right\|_{\infty} \rightarrow 0 \text { as } s \rightarrow \infty .
$$

Hence the following lemma holds.

Lemma 4.4. Let $y \in \mathcal{V}^{(1)}(\bar{x}), w \in \mathcal{V}^{(2)}(\bar{x}, y)$ and $h_{s} \rightarrow 0+$ be a sequence of reals. Then there exists a sequence of elements $x_{s} \in S, s=1, \ldots$ satisfying (4.7).

4.2. Incompatibility of a second order system. Fix a strong local minimizer $(\bar{x}, \bar{u})$. Without loss of generality, we may assume that

$$
g_{0}(\bar{x}(1))=0, \quad \bar{x}(1) \in \partial K_{i}^{f}, \quad i=1, \ldots, k,
$$

and

$$
\mathcal{M}_{j 0} \neq \emptyset, \quad j=1, \ldots, q .
$$

In the other words, we assume that all constraints are active at $\bar{x}$. It follows that $I_{g}=\{1, \ldots, k\}$.

Lemma 4.5. Let $\xi=(y, u) \in \mathcal{C}_{0}(\bar{x}, \bar{u})$ and $V^{2}(\bar{u}, u) \neq \emptyset$. Then the system of conditions

$$
\begin{aligned}
& g_{i}^{\prime}(\bar{x}(1)) w(1)+\frac{1}{2}\left\langle g_{i}^{\prime \prime}(\bar{x}(1)) y(1), y(1)\right\rangle<0, \quad i=0, \ldots, k, \\
& \max _{t \in \mathcal{M}_{j 0}}\left(\Phi_{j}^{\prime}(\bar{x}(t)) w(t)+\frac{1}{2}\left\langle\Phi_{j}^{\prime \prime}(\bar{x}(t)) y(t), y(t)\right\rangle\right)<0, \quad j=1, \ldots, q,
\end{aligned}
$$




$$
w \in \mathcal{V}^{(2)}(\bar{x}, y)
$$

is incompatible.

Proof Indeed, by Lemma 4.1, condition $(y, u) \in \mathcal{C}_{0}(\bar{x}, \bar{u})$ implies that $y \in \mathcal{V}^{(1)}(\bar{x})$. Assume for a moment that there exists an element $w$ satisfying (4.10)-(4.12). Consider any sequence of reals $h_{s} \rightarrow 0+$. Then, by Lemma 4.4, there exists a sequence $x_{s} \in S$ as in (4.7). Let $u_{s}$ be such that $\left(x_{s}, u_{s}\right)$ solves (1.3). Then, for any $i=0,1, \ldots, k$,

$$
\begin{aligned}
g_{i}\left(x_{s}(1)\right) & =g_{i}\left(\bar{x}(1)+h_{s} y(1)+h_{s}^{2} w(1)+h_{s}^{2} r_{s}(1)\right)=g_{i}(\bar{x}(1))+h_{s} g_{i}^{\prime}(\bar{x}(1)) y(1) \\
& +h_{s}^{2}\left(g_{i}^{\prime}(\bar{x}(1)) w(1)+\frac{1}{2}\left\langle g_{i}^{\prime \prime}(\bar{x}(1)) y(1), y(1)\right\rangle\right)+o\left(h_{s}^{2}\right) .
\end{aligned}
$$

Taking into account (4.8), (3.8), and (4.10), we deduce that for some $\varepsilon>0$ and for all $s$ large enough, the inequality

$$
g_{i}\left(x_{s}(1)\right) \leq-\varepsilon h_{s}^{2}+o\left(h_{s}^{2}\right)
$$

is fulfilled. Similarly, for any $j=1, \ldots, q$, we have

$$
\begin{aligned}
& \Phi_{j}\left(x_{s}(t)\right)=\Phi_{j}\left(\bar{x}(t)+h_{s} y(t)+h_{s}^{2} w(t)+h_{s}^{2} r_{s}(t)\right)= \\
& \Phi_{j}(\bar{x}(t))+h_{s} \Phi_{j}^{\prime}(\bar{x}(t)) y(t)+h_{s}^{2}\left(\Phi_{j}^{\prime}(\bar{x}(t)) w(t)+\frac{1}{2}\left\langle\Phi_{j}^{\prime \prime}(\bar{x}(t)) y(t), y(t)\right\rangle\right) \\
& +h_{s}^{2} \zeta_{s}(t), \quad \text { where }\left\|\zeta_{s}\right\|_{\infty} \rightarrow 0 \text { as } s \rightarrow \infty .
\end{aligned}
$$

Since $\Phi_{j}^{\prime}(\bar{x}(\cdot)) w(\cdot)+\frac{1}{2}\left\langle\Phi_{j}^{\prime \prime}(\bar{x}(\cdot)) y(\cdot), y(\cdot)\right\rangle$ is continuous, by $(4.11)$, there exists $\delta>0$ such that the strict inequality

$$
\Phi_{j}^{\prime}(\bar{x}(t)) w(t)+\frac{1}{2}\left\langle\Phi_{j}^{\prime \prime}(\bar{x}(t)) y(t), y(t)\right\rangle<0
$$

is fulfilled also on the set $\mathcal{M}_{j \delta}$. Taking, if necessary, a smaller $\delta>0$, we obtain, according (3.13), that $\Phi_{j}^{\prime}(\bar{x}(t)) y(t) \leq 0$ on $\mathcal{M}_{j \delta}$. Since $\Phi_{j}(\bar{x}(t)) \leq 0$ on $[0,1]$, it follows from (4.14) that for some $\varepsilon>0$ and for all small enough $\delta>0$, we have

$$
\max _{t \in \mathcal{M}_{j \delta}} \Phi_{j}\left(x_{s}(t)\right) \leq-\varepsilon h_{s}^{2}+o\left(h_{s}^{2}\right) .
$$

Further, on the set $[0,1] \backslash \mathcal{M}_{j \delta}$ we have either $\Phi_{j}(\bar{x}(t))<-\delta$, or $d_{\partial K_{j}}(\bar{x}(t))>\delta$, and in the both cases

$$
\sup _{t \in[0,1] \backslash \mathcal{M}_{j \delta}} \Phi_{j}\left(x_{s}(t)\right) \leq 0
$$

for all $s$ large enough. Hence for all large $s$,

$$
\max _{t \in[0,1]} \Phi_{j}\left(x_{s}(t)\right) \leq 0 .
$$

The existence of the sequence $\left(x_{s}, u_{s}\right)$ which solves (1.3), satisfies (4.13) and (4.15), and such that $\left\|x_{s}-\bar{x}\right\|_{\infty} \rightarrow 0$ as $s \rightarrow \infty$, implies that $(\bar{x}, \bar{u})$ is not a point of a strong local minimum. The obtained contradiction ends the proof.

Fix $\xi=(y, u) \in \mathcal{C}_{0}(\bar{x}, \bar{u})$ with $V^{2}(\bar{u}, u) \neq \emptyset$. For any $i=0, \ldots, k$, define the following open, possibly empty, affine half-space in the space $\left.C([0,1]), \mathbb{R}^{n}\right)$ :

$$
\left.\mathcal{Q}_{i}=\left\{w \in C([0,1]), \mathbb{R}^{n}\right): g_{i}^{\prime}(\bar{x}(1)) w(1)+\frac{1}{2}\left\langle g_{i}^{\prime \prime}(\bar{x}(1)) y(1), y(1)\right\rangle<0\right\} .
$$


Note that $\mathcal{Q}_{i}$ is empty if and only if $g_{i}^{\prime}(\bar{x}(1))=0$ and $\left\langle g_{i}^{\prime \prime}(\bar{x}(1)) y(1), y(1)\right\rangle \geq 0$. For any $j=1, \ldots, q$, define an open convex, possibly empty, subset of $\left.C([0,1]), \mathbb{R}^{n}\right)$

$$
\left.\mathcal{F}_{j}=\left\{w \in C([0,1]), \mathbb{R}^{n}\right): \max _{t \in \mathcal{M}_{j 0}}\left(\Phi_{j}^{\prime}(\bar{x}(t)) w(t)+\frac{1}{2}\left\langle\Phi_{j}^{\prime \prime}(\bar{x}(t)) y(t), y(t)\right\rangle\right)<0\right\} .
$$

Note that $\mathcal{F}_{j}$ is empty if and only if $\Phi_{j}^{\prime}(\bar{x}(t))=0$ and $\left\langle\Phi_{j}^{\prime \prime}(\bar{x}(t)) y(t), y(t)\right\rangle \geq 0$ for some $t \in \mathcal{M}_{j 0}$.

It follows from Lemmas 4.2 and 4.5 that $\mathcal{V}^{(2)}(\bar{x}, y) \neq \emptyset$ and that

$$
\left(\bigcap_{i=0}^{k} \mathcal{Q}_{i}\right) \bigcap\left(\bigcap_{j=1}^{q} \mathcal{F}_{j}\right) \bigcap \imath\left(\tilde{\mathcal{V}}^{(2)}(\bar{x}, y)\right)=\emptyset .
$$

Hence this condition is necessary for $(\bar{x}, \bar{u})$ to be a strong local minimizer. Recall that this condition was written for an arbitrary fixed pair $\xi=(y, u) \in \mathcal{C}_{0}(\bar{x}, \bar{u})$ with $V^{2}(\bar{u}, u) \neq \emptyset$. Since $\mathcal{Q}_{i}$ and $\mathcal{F}_{j}$ are open, (4.16) implies

$$
\left(\bigcap_{i=0}^{k} Q_{i}\right) \bigcap\left(\bigcap_{j=1}^{q} \mathcal{F}_{j}\right) \bigcap \mathcal{E}^{2}=\emptyset,
$$

where the nonempty convex (by Lemma 4.2) closed set $\mathcal{E}^{2}$ was defined by (4.6). Now Theorem 2.1 can be applied.

4.3. Application of Theorem 2.1. We analyze next the necessary optimality condition (4.17) in the light of Theorem 2.1. First of all, let us establish the correspondence between notation of condition (4.17) and Theorem 2.1: define

$X=C\left([0,1], \mathbb{R}^{n}\right)$,

$l_{i}: w(\cdot) \rightarrow g_{i}^{\prime}(\bar{x}(1)) w(1), m_{i}=\frac{1}{2}\left\langle g_{i}^{\prime \prime}(\bar{x}(1)) y(1), y(1)\right\rangle, i=0, \ldots, k$,

$Z_{j}=C([0,1], \mathbb{R}), \varphi_{j}: z(\cdot) \rightarrow \max _{t \in \mathcal{M}_{j 0}} z(t), j=1, \ldots, q$,

$P_{j}: w(\cdot) \rightarrow \Phi_{j}^{\prime}(\bar{x}(\cdot)) w(\cdot), z_{j}(\cdot)=\frac{1}{2}\left\langle\Phi_{j}^{\prime \prime}(\bar{x}(\cdot)) y(\cdot), y(\cdot)\right\rangle, j=1, \ldots, q$.

According to Theorem 2.1 and Lemma 2.6, there exist reals $\alpha_{i} \geq 0, i=0, \ldots, k$, $\beta_{j} \geq 0, j=1, \ldots, q$, probability measures $\tilde{\mu}_{j}$ with supp $\tilde{\mu}_{j} \subset \mathcal{M}_{0 j}, j=1, \ldots, q$, and $z^{*} \in C\left([0,1], \mathbb{R}^{n}\right)^{*}$ such that the following three conditions hold:

$$
\sum_{i=0}^{k} \alpha_{i}+\sum_{j=1}^{q} \beta_{j}=1,
$$

$$
\begin{gathered}
\sum_{i=0}^{k} \alpha_{i} g_{i}^{\prime}(\bar{x}(1)) w(1)+\sum_{j=1}^{q} \beta_{j} \int_{[0,1]} \Phi_{j}^{\prime}(\bar{x}(t)) w(t) \mathrm{d} \tilde{\mu}_{j}=\left\langle z^{*}, w\right\rangle \quad \forall w \in C\left([0,1], \mathbb{R}^{n}\right), \\
\frac{1}{2} \sum_{i=0}^{k} \alpha_{i}\left\langle g_{i}^{\prime \prime}(\bar{x}(1)) y(1), y(1)\right\rangle+\frac{1}{2} \sum_{j=1}^{q} \beta_{j} \int_{[0,1]}\left\langle\Phi_{j}^{\prime \prime}(\bar{x}(t)) y(t), y(t)\right\rangle \mathrm{d} \tilde{\mu}_{j}+\inf \left\langle z^{*}, \mathcal{E}^{2}\right\rangle \geq 0 .
\end{gathered}
$$

Replacing $\sum_{i=0}^{k} \alpha_{i} g_{i}^{\prime}(\bar{x}(1))$ and $\sum_{i=0}^{k} \alpha_{i} g_{i}^{\prime \prime}(\bar{x}(1))$ by $l_{x}(\bar{x}(1), \alpha)$ and $l_{x x}(\bar{x}(1), \alpha)$, respectively, and each product $\beta_{j} \mathrm{~d} \tilde{\mu}_{j}$ by $\mathrm{d} \mu_{j}, j=1, \ldots, q$, and also using the obvious 
equality $\inf \left\langle z^{*}, \mathcal{E}^{2}\right\rangle=\inf \left\langle z^{*}, \imath\left(\tilde{\mathcal{V}}^{(2)}(\bar{x}, y)\right)\right\rangle$, we get the following system of conditions

$$
\begin{gathered}
\sum_{i=0}^{k} \alpha_{i}+\sum_{j=1}^{q} \mu_{j}([0,1])=1, \\
(4.18) \quad l_{x}(\bar{x}(1), \alpha) w(1)+\sum_{j=1}^{q} \int_{[0,1]} \Phi_{j}^{\prime}(\bar{x}(t)) w(t) \mathrm{d} \mu_{j}=\left\langle z^{*}, w\right\rangle \quad \forall w \in C\left([0,1], \mathbb{R}^{n}\right), \\
(4.19) \quad \\
\frac{1}{2}\left\langle l_{x x}(\bar{x}(1), \alpha) y(1), y(1)\right\rangle+\frac{1}{2} \sum_{j=1}^{q} \int_{[0,1]}\left\langle\Phi_{j}^{\prime \prime}(\bar{x}(t)) y(t), y(t)\right\rangle \mathrm{d} \mu_{j}+\inf \left\langle z^{*}, \imath\left(\tilde{\mathcal{V}}^{(2)}(\bar{x}, y)\right)\right\rangle \geq 0 .
\end{gathered}
$$

Let the right-continuous function of bounded variation $p$ solve the adjoint system (3.3), (3.4). For $v \in V^{2}(\bar{u}, u)$ (see (3.15)), $\eta \in K$ (see (4.2)) consider the solution $w$ of (4.3). Then

$$
\begin{aligned}
& \mathrm{d}(p(t) w(t))=-p(t) f_{x}[t] w(t) \mathrm{d} t-\sum_{j=1}^{q} \Phi_{j}^{\prime}(\bar{x}(t)) w(t) \mathrm{d} \mu_{j}(t) \\
& +p(t)\left(f_{x}[t] w(t)+f_{u}[t] v(t)+\frac{1}{2} f^{\prime \prime}[t](\xi(t), \xi(t))+\eta(t)\right) \mathrm{d} t \\
& =-\sum_{j=1}^{q} \Phi_{j}^{\prime}(\bar{x}(t)) w(t) \mathrm{d} \mu_{j}(t)+p(t)(\beta(t)+\eta(t)) \mathrm{d} t
\end{aligned}
$$

where

$$
\beta(t)=f_{u}[t] v(t)+\frac{1}{2} f^{\prime \prime}[t](\xi(t), \xi(t))
$$

Integrating the obtained equality over the interval $[0,1]$ and taking into account that $w(0)=0$, we get

$$
p(1) w(1)=-\sum_{j=1}^{q} \int_{[0,1]} \Phi_{j}^{\prime}(\bar{x}(t)) w(t) \mathrm{d} \mu_{j}+\int_{0}^{1} p(t)(\beta(t)+\eta(t)) \mathrm{d} t,
$$

or, equivalently,

$$
l_{x}(\bar{x}(1), \alpha) w(1)+\sum_{j=1}^{q} \int_{[0,1]} \Phi_{j}^{\prime}(\bar{x}(t)) w(t) \mathrm{d} \mu_{j}=\int_{0}^{1} p(t)(\beta(t)+\eta(t)) \mathrm{d} t .
$$

Using (4.18), we obtain $\left\langle z^{*}, w\right\rangle=\int_{0}^{1} p(t)(\beta(t)+\eta(t)) \mathrm{d} t$. Consequently, by the definition of $\beta$,

$$
\left\langle z^{*}, w\right\rangle=\int_{0}^{1} \mathcal{H}_{u}[t] v(t) \mathrm{d} t+\frac{1}{2} \int_{0}^{1}\left\langle\mathcal{H}^{\prime \prime}[t] \xi(t), \xi(t)\right\rangle \mathrm{d} t+\int_{0}^{1} p(t) \eta(t) \mathrm{d} t .
$$

Recall that here $\eta \in K$ and $v \in V^{2}(\bar{u}, u)$. Then, according to the definition of $\tilde{\mathcal{V}}^{(2)}(\bar{x}, y)$ (see (4.4)) and inequality (4.19), we get

$$
\Omega(\xi, \alpha, p, \mu)+\inf _{v \in V^{2}(\bar{u}, u)} \int_{0}^{1} \mathcal{H}_{u}[t] v(t) \mathrm{d} t+\inf _{\eta \in K} \int_{0}^{1} p(t) \eta(t) \mathrm{d} t \geq 0
$$


where $\Omega$ is defined by (3.17). Since $K$ is a cone, it follows that

$$
\begin{gathered}
\Omega(\xi, \alpha, p, \mu)+\inf _{v \in V^{2}(\bar{u}, u)} \int_{0}^{1} \mathcal{H}_{u}[t] v(t) \mathrm{d} t \geq 0, \\
\int_{0}^{1} p(t) \eta(t) \mathrm{d} t \geq 0 \quad \forall \eta \in K .
\end{gathered}
$$

We have shown in [10, Proof of Theorem 3.2] that (4.21) implies the minimum principle $(3.5)$ and hence $(\alpha, p, \mu) \in M(\bar{x}, \bar{u})$. Moreover we have shown there that (4.20) yields (3.18). Theorem 3.3 is completely proved.

Remark 4.1 (Normality of necessary conditions). The above proof and Theorem 2.1 imply that in Theorem $3.3 \alpha_{0}>0$ whenever there exists $w \in \tilde{\mathcal{V}}^{(2)}(\bar{x}, y)$ such that

$$
g_{i}^{\prime}(\bar{x}(1)) w(1)+\frac{1}{2}\left\langle g_{i}^{\prime \prime}(\bar{x}(1)) y(1), y(1)\right\rangle<0, \quad i=1, \ldots, k,
$$

and

$$
\max _{t \in \mathcal{M}_{j 0}}\left(\Phi_{j}^{\prime}(\bar{x}(t)) w(t)+\frac{1}{2}\left\langle\Phi_{j}^{\prime \prime}(\bar{x}(t)) y(t), y(t)\right\rangle\right)<0, \quad j=1, \ldots, q
$$

5. Some special cases of $U$. In this section we consider problem (1.1)-(1.4) with the set $U$ having a special structure. Then the statement of the main result can be simplified.

5.1. The case when $U$ is a union of convex polytopes. As usual, we understand a convex polytope as the convex hull of a finite number of points in $\mathbb{R}^{m}$. Consider problem (1.1)-(1.4) with the set $U$ being a union of convex polytopes $U_{k} \subset \mathbb{R}^{m}, k=1, \ldots, r$. Then $U$ is compact and in general, it is not convex.

Denote by ext $U_{k}$ the set of all the extremal points of $U_{k}$. Let an admissible pair $(\bar{x}, \bar{u})$ affords a strong local minimum in our problem with $U$ as above and assume that

$$
\bar{u}(t) \in \bigcup_{k=1}^{r} \operatorname{ext} U_{k} \quad \text { a.e. in } \quad[0,1]
$$

Then it is not difficult to realise that $\mathcal{C}_{0}(\bar{x}, \bar{u})=\mathcal{C}_{\Phi}(\bar{x}, \bar{u})$. Let $\xi=(y, u) \in \mathcal{C}_{\Phi}(\bar{x}, \bar{u})$. Then condition (3.18) holds with some $(\alpha, p, \mu) \in M(\bar{x}, \bar{u})$. Moreover, it can be verified, similarly to [10], that the function $\Upsilon(u(\cdot), p(\cdot))$ appearing in the necessary condition (3.18) is equal to zero. Thus the following theorem holds.

THEOREM 5.1. Let $U$ be a union of convex polytopes, and let $(\bar{x}, \bar{u})$ be a strong local minimizer of problem (1.1)-(1.4) with $\bar{u}$ satisfying condition (5.1). Then, for any $\xi=(y, u) \in \mathcal{C}_{\Phi}(\bar{x}, \bar{u})$, there exists $(\alpha, p, \mu) \in M(\bar{x}, \bar{u})$, such that

$\left\langle l_{x x}(\bar{x}(1), \alpha) y(1), y(1)\right\rangle+\int_{0}^{1}\left\langle\mathcal{H}^{\prime \prime}[t] \xi(t), \xi(t)\right\rangle \mathrm{d} t+\sum_{j=1}^{q} \int_{[0,1]}\left\langle\Phi_{j}^{\prime \prime}(\bar{x}(t)) y(t), y(t)\right\rangle \mathrm{d} \mu_{j} \geq 0$. 
5.2. The case when $U$ is given by inequalities. Consider now problem (1.1)(1.4), with

$$
U=\left\{u \in \mathbb{R}^{m}: \varphi_{i}(u) \leq 0, \quad i=1, \ldots, r\right\},
$$

where $\varphi_{i}: \mathbb{R}^{m} \rightarrow \mathbb{R}$ are $C^{2}$ and at each point $u \in \partial U$ the gradients $\varphi_{i}^{\prime}(u), i \in$ $I_{\varphi}(u)$ are positively independent (i.e., independent with nonnegative coefficients in the definition), where $I_{\varphi}(u)=\left\{i: \varphi_{i}(u)=0\right\}$ is the set of active indices at $u$ and $\varphi=\left(\varphi_{1}, \ldots, \varphi_{r}\right)$. We also assume that $U$ is compact. By [2, Chapter 4], for every $u_{0} \in U$,

$$
T_{U}^{b}\left(u_{0}\right)=\left\{u \in \mathbb{R}^{m}: \varphi_{i}^{\prime}\left(u_{0}\right) u \leq 0, i \in I_{\varphi}\left(u_{0}\right)\right\} .
$$

Such control set $U$ was also considered in $[9$, Sec. 7] for the Mayer problem in the absence of state and final-point constraints. Here we pay attention only to the changes which are introduced due to the presence of these constraints.

Let $(\bar{x}, \bar{u}) \in \Xi$ be a strong local minimizer in problem (1.1)-(1.4), and hence (3.1)(3.6) are satisfied for some $(\alpha, p, \mu)$. From [9, Section 5] we know that there exists a measurable essentially bounded function $\lambda(\cdot)=\left(\lambda_{1}(\cdot), \ldots, \lambda_{r}(\cdot)\right)$ such that

$$
\begin{gathered}
\lambda_{1}(t) \geq 0, \ldots, \lambda_{r}(t) \geq 0, \quad \lambda_{i}(t) \varphi_{i}(\bar{u}(t))=0, \quad \text { a.e. }, \quad i=1, \ldots, r, \\
\mathcal{H}_{u}(\bar{x}(t), \bar{u}(t), p(t))+\sum_{i=1}^{r} \lambda_{i}(t) \varphi_{i}^{\prime}(\bar{u}(t))=0 \quad \text { a.e. }
\end{gathered}
$$

The augmented Hamiltonian is defined by $\mathcal{H}^{a}: \mathbb{R}^{n} \times \mathbb{R}^{m} \times \mathbb{R}^{n} \times \mathbb{R}^{r} \rightarrow \mathbb{R}$,

$$
\mathcal{H}^{a}(x, u, p, \lambda)=\mathcal{H}(x, u, p)+\lambda \varphi(u),
$$

where $\lambda=\left(\lambda_{1}, \ldots, \lambda_{r}\right)$. Then (5.3) yields $\mathcal{H}_{u}^{a}(\bar{x}(t), \bar{u}(t), p(t), \lambda(t))=0$ a.e.

Define the set of multipliers

$M^{a}(\bar{x}, \bar{u})=\left\{(\alpha, p, \lambda, \mu):(\alpha, p, \mu) \in M(\bar{x}, \bar{u}), \lambda \in L^{\infty}\left([0,1], \mathbb{R}^{r}\right)\right.$, and (5.2), (5.3) hold $\}$.

Consider the critical cone $\mathcal{C}(\bar{x}, \bar{u})$, defined by (3.8)-(3.11), and set

$$
\mathcal{M}_{i 0}^{\varphi}=\left\{t \in[0,1]: \bar{u}(t) \in \partial U, \varphi_{i}(\bar{u}(t))=0\right\}, \quad i=1, \ldots, r .
$$

Then (3.11) is equivalent to

$$
\varphi_{i}^{\prime}(\bar{u}(t)) u(t) \leq 0 \quad \text { a.e. in } \quad \mathcal{M}_{i 0}^{\varphi}, \quad i=1, \ldots, r .
$$

Hence

Proposition 5.2. The critical cone $\mathcal{C}(\bar{x}, \bar{u})$ is defined by conditions (3.8)-(3.10) and (5.4).

For any $\delta>0$, define

$$
\mathcal{M}_{i \delta}^{\varphi}=\left\{t \in[0,1]: \mathrm{d}_{\partial U}(\bar{u}(t)) \leq \delta, \varphi_{i}(\bar{u}(t)) \geq-\delta\right\}, \quad i=1, \ldots, r .
$$

Consider $u(\cdot) \in L^{\infty}\left([0,1], \mathbb{R}^{m}\right)$ satisfying:

$$
\exists \delta>0 \text { such that } \varphi_{i}^{\prime}(\bar{u}(t)) u(t) \leq 0 \quad \text { a.e. on } \mathcal{M}_{i \delta}^{\varphi}, \quad i=1, \ldots, r \text {. }
$$


Lemma 5.3. Condition (5.5) implies condition (3.12).

The proof is given in [10, Lemma 7.2].

Now consider the function $\Upsilon(u(\cdot), p(\cdot))$ as in (3.16). It was proved in $[9$, Section $5]$ that the set $V^{2}(\bar{u}, u)$ is nonempty, and that $\lambda$ can be chosen in such a way that it is essentially bounded, (5.2) and (5.3) are satisfied and

$$
\Upsilon(u(t), p(t))=\frac{1}{2} \sum_{i=1}^{r} \lambda_{i}(t)\left\langle\varphi_{i}^{\prime \prime}(\bar{u}(t)) u(t), u(t)\right\rangle \quad \text { a.e. }
$$

Define

$$
\begin{aligned}
\Omega^{a}(\xi, \alpha, p, \lambda, \mu):= & \frac{1}{2}\left\langle l_{x x}(\bar{x}(1), \alpha) y(1), y(1)\right\rangle+\frac{1}{2} \int_{0}^{1}\left\langle\left(\mathcal{H}^{a}\right)^{\prime \prime}[t] \xi(t), \xi(t)\right\rangle \mathrm{d} t \\
& +\frac{1}{2} \sum_{j=1}^{q} \int_{[0,1]}\left\langle\Phi_{j}^{\prime \prime}(\bar{x}(t)) y(t), y(t)\right\rangle \mathrm{d} \mu_{j} .
\end{aligned}
$$

From Theorem 3.3 we deduce

TheOrem 5.4. Let $(\bar{x}, \bar{u})$ be a strong local minimizer. Then $M^{a}(\bar{x}, \bar{u}) \neq \emptyset$ and for any $\xi=(y, u) \in \mathcal{C}(\bar{x}, \bar{u})$ satisfying (5.5) and (3.13), there exists $(\alpha, p, \lambda, \mu) \in M^{a}(\bar{x}, \bar{u})$ such that $\Omega^{a}(\xi, \alpha, p, \lambda, \mu) \geq 0$.

\section{Appendix.}

6.1. Proof of Lemma 2.3. Let $x^{*} \in M^{d}$. If $x^{*}=0$, we take $\alpha=0$ and choose $x_{1}^{*} \in \operatorname{dom} F^{*}$ arbitrary. So let $0 \neq x^{*} \in M^{d}$ and set $\inf \left\langle x^{*}, M\right\rangle=\gamma$. Since $M$ is open, for any $x \in M$ we have $\left\langle x^{*}, x\right\rangle>\gamma$. In the space $X \times \mathbb{R}$ consider two sets

$$
\Omega_{0}:=\{(x, t) \in X \times \mathbb{R}: F(x)<t\}, \quad \Omega_{1}:=\left\{(x, t) \in X \times \mathbb{R}:\left\langle x^{*}, x\right\rangle \leq \gamma, t=0\right\} .
$$

Both sets are convex and the first set is open. Moreover $\Omega_{0} \cap \Omega_{1}=\emptyset$. By the separation theorem, there exist $x_{1}^{*} \in X^{*}, \beta \in \mathbb{R}$, not vanishing simultaneously, and a constant $c \in \mathbb{R}$ such that $l\left(\Omega_{0}\right)<c$ and $l\left(\Omega_{1}\right) \geq c$, where $l(x, t)=\left\langle x_{1}^{*}, x\right\rangle+\beta t$ for $x \in X, t \in \mathbb{R}$. It means that

$$
\begin{gathered}
F(x)<t \quad \Rightarrow \quad\left\langle x_{1}^{*}, x\right\rangle+\beta t<c, \\
\left\langle x^{*}, x\right\rangle \leq \gamma \quad \Rightarrow \quad\left\langle x_{1}^{*}, x\right\rangle \geq c .
\end{gathered}
$$

From (6.1) it follows that $\beta \leq 0$. But if $\beta=0$, then $x_{1}^{*} \neq 0$ and from (6.1) we get $\left\langle x_{1}^{*}, x\right\rangle<c$ for every $x \in X$, which is impossible. Therefore, $\beta<0$ and then we can take the pair $\left(x_{1}^{*}, \beta\right)$ such that $\beta=-1$. Then condition (6.1) becomes

$$
F(x)<t \quad \Rightarrow \quad\left\langle x_{1}^{*}, x\right\rangle-t<c .
$$

Further, (6.2) implies that there exists $\alpha>0$ satisfying $x^{*}=-\alpha x_{1}^{*}, \gamma \leq-\alpha c$, and from (6.3) we easily get $\left\langle x_{1}^{*}, x\right\rangle-F(x) \leq c$ for all $x \in X$. Thus $x_{1}^{*} \in \operatorname{dom} F^{*}$ and $F^{*}\left(x_{1}^{*}\right) \leq c$. Consequently, $-\alpha F^{*}\left(x_{1}^{*}\right) \geq-\alpha c \geq \gamma$, and then $\inf \left\langle x^{*}, M\right\rangle \leq-\alpha F^{*}\left(x_{1}^{*}\right)$.

Now we establish the opposite inequality. Since $x_{1}^{*} \in \operatorname{dom} F^{*}$, we have $\left\langle x_{1}^{*}, x\right\rangle-$ $F^{*}\left(x_{1}^{*}\right) \leq F(x)$ for all $x \in X$. This implies that $\left\langle x_{1}^{*}, x\right\rangle-F^{*}\left(x_{1}^{*}\right)<0$ for all $x \in M$. Multiplying by $\alpha>0$, we obtain $\alpha\left\langle x_{1}^{*}, x\right\rangle-\alpha F^{*}\left(x_{1}^{*}\right)<0$ for all $x \in M$. Consequently, $\left\langle x^{*}, x\right\rangle>-\alpha F^{*}\left(x_{1}^{*}\right)$ for all $x \in M$. This implies that $\inf \left\langle x^{*}, M\right\rangle \geq-\alpha F^{*}\left(x_{1}^{*}\right)$. 
6.2. Proof of Lemma 2.5. The continuity and convexity of the function $F(x)=$ $\varphi\left(y_{0}+A x\right)$ follow from the same properties of $\varphi$ and the continuity of the operator $A$. Let us show, that if $y^{*} \in \operatorname{dom} \varphi^{*}$ and $x^{*}=A^{*} y^{*}$, then $x^{*} \in \operatorname{dom} F^{*}$ and

$$
F^{*}\left(x^{*}\right) \leq \varphi^{*}\left(y^{*}\right)-\left\langle y^{*}, y_{0}\right\rangle .
$$

Indeed, if $y^{*} \in \operatorname{dom} \varphi^{*}$, then $\left\langle y^{*}, y\right\rangle-\varphi(y) \leq \varphi^{*}\left(y^{*}\right)<+\infty$ for all $y \in Y$, and therefore

$$
F(x)=\varphi\left(A x+y_{0}\right) \geq\left\langle y^{*}, y_{0}+A x\right\rangle-\varphi^{*}\left(y^{*}\right) \quad \forall x \in X .
$$

Let $x^{*}=A^{*} y^{*}$. Then

$$
\left\langle x^{*}, x\right\rangle-F(x) \leq\left\langle x^{*}, x\right\rangle+\varphi^{*}\left(y^{*}\right)-\left\langle y^{*}, y_{0}+A x\right\rangle=\varphi^{*}\left(y^{*}\right)-\left\langle y^{*}, y_{0}\right\rangle \quad \forall x \in X .
$$

The condition $x^{*} \in \operatorname{dom} F^{*}$ and inequality (6.4) follow.

It remains to prove that if $x^{*} \in \operatorname{dom} F^{*}$, then there exists $y^{*} \in \operatorname{dom} \varphi^{*}$ such that $x^{*}=A^{*} y^{*}$ and

$$
F^{*}\left(x^{*}\right) \geq \varphi^{*}\left(y^{*}\right)-\left\langle y^{*}, y_{0}\right\rangle .
$$

To this end, in the space $Z=X \times Y \times \mathbb{R}$ define three sets:

$$
\begin{aligned}
& M=\left\{(x, y, t) \in Z: y=A x+y_{0}\right\}, M_{0}=\{(x, y, t) \in Z: \varphi(y)<t\}, \\
& M_{1}=\left\{(x, y, t) \in Z:\left\langle x^{*}, x\right\rangle-F^{*}\left(x^{*}\right)>t\right\} .
\end{aligned}
$$

Each set is nonempty, convex and moreover $M_{0}$ and $M_{1}$ are open. We claim that,

$$
M_{0} \cap M_{1} \cap M=\emptyset .
$$

Indeed, if $(x, y, t) \in M_{0} \cap M_{1} \cap M$, then

$$
y=A x+y_{0}, \quad \varphi(y)<t, \quad\left\langle x^{*}, x\right\rangle-F^{*}\left(x^{*}\right)>t .
$$

Thus,

$$
\left\langle x^{*}, x\right\rangle-F^{*}\left(x^{*}\right)>\varphi(y)=\varphi\left(A x+y_{0}\right)=F(x),
$$

whence $\left\langle x^{*}, x\right\rangle-F^{*}\left(x^{*}\right)>F(x)$, which is impossible. Therefore, condition (6.6) is fulfilled. Also note, that $M \cap M_{0} \neq \emptyset$.

By Lemma 2.2, there exist $l_{M} \in M^{d}, l_{0} \in M_{0}^{d}, l_{1} \in M_{1}^{d}$, not all equal to zero, and such that

$$
\begin{gathered}
l_{M}+l_{0}+l_{1}=0 \\
\inf l_{M}(M)+\inf l_{0}\left(M_{0}\right)+\inf l_{1}\left(M_{1}\right) \geq 0 .
\end{gathered}
$$

Since $M \cap M_{0} \neq \emptyset$, from Lemma 2.2 we deduce that

$$
l_{1} \neq 0 .
$$

Set

$$
\gamma_{M}=\inf l_{M}(M), \quad \gamma_{0}=\inf l_{0}\left(M_{0}\right), \quad \gamma_{1}=\inf l_{1}\left(M_{1}\right),
$$


and let $x_{M}^{*}, x_{0}^{*}, x_{1}^{*} \in X^{*}, y_{M}^{*}, y_{0}^{*}, y_{1}^{*} \in Y^{*}, \beta_{M}, \beta_{0}, \beta_{1} \in \mathbb{R}$ be so that

$$
l_{M}(z)=\left\langle x_{M}^{*}, x\right\rangle+\left\langle y_{M}^{*}, y\right\rangle+\beta_{M} t, \quad l_{i}(z)=\left\langle x_{i}^{*}, x\right\rangle+\left\langle y_{i}^{*}, y\right\rangle+\beta_{i} t, \quad i=0,1
$$

for all $z=(x, y, t) \in Z$. Then condition (6.7) implies

$$
x_{M}^{*}+x_{0}^{*}+x_{1}^{*}=0, \quad y_{M}^{*}+y_{0}^{*}+y_{1}^{*}=0, \quad \beta_{M}+\beta_{0}+\beta_{1}=0,
$$

and from (6.8) and (6.10) we get

$$
\gamma_{M}+\gamma_{0}+\gamma_{1} \geq 0
$$

Further, from the definitions of the sets $M, M_{0}, M_{1}$ it follows that

$$
\begin{gathered}
y=A x+y_{0} \quad \Rightarrow \quad\left\langle x_{M}^{*}, x\right\rangle+\left\langle y_{M}^{*}, y\right\rangle+\beta_{M} t \geq \gamma_{M}, \\
\varphi(y)<t \quad \Rightarrow \quad\left\langle x_{0}^{*}, x\right\rangle+\left\langle y_{0}^{*}, y\right\rangle+\beta_{0} t \geq \gamma_{0}, \\
\left\langle x^{*}, x\right\rangle-F^{*}\left(x^{*}\right)>t \quad \Rightarrow \quad\left\langle x_{1}^{*}, x\right\rangle+\left\langle y_{1}^{*}, y\right\rangle+\beta_{1} t>\gamma_{1} .
\end{gathered}
$$

The latter inequality is strict since $l_{1} \neq 0$ and the set $M_{1}$ is open. In view of (6.13),

$$
\left\langle x_{M}^{*}, x\right\rangle+\left\langle y_{M}^{*}, A x+y_{0}\right\rangle+\beta_{M} t \geq \gamma_{M} \quad \forall x \in X, \quad \forall t \in \mathbb{R} .
$$

Therefore

$$
x_{M}^{*}+A^{*} y_{M}^{*}=0, \quad \beta_{M}=0, \quad \gamma_{M}-\left\langle y_{M}^{*}, y_{0}\right\rangle \leq 0,
$$

and then by the third relation in (6.11), $\beta_{1}=-\beta_{0}$. Further, (6.14) implies $x_{0}^{*}=$ $0, \beta_{0} \geq 0$, and then by the first relation in (6.11), $x_{M}^{*}=-x_{1}^{*}$. From (6.15) we get $y_{1}^{*}=0$, and therefore (6.15) takes the form

$$
\left\langle x^{*}, x\right\rangle-t>F^{*}\left(x^{*}\right) \quad \Rightarrow \quad\left\langle x_{1}^{*}, x\right\rangle+\beta_{1} t>\gamma_{1},
$$

whence

$$
x_{1}^{*}=\alpha x^{*}, \quad \beta_{1}=-\alpha \quad \text { for some } \alpha \geq 0 .
$$

If $\alpha=0$, then $x_{1}^{*}=0, y_{1}^{*}=0$, and $\beta_{1}=0$, whence $l_{1}=0$ which contradicts (6.9). Therefore $\alpha>0$, and then we can take $\alpha=1$, because conditions (6.7) and (6.8) are homogeneous with respect to $l_{0}, l_{1}, l_{M}$. Then $x^{*}=x_{1}^{*}=-x_{M}^{*}, \beta_{1}=-1$, and hence $\beta_{0}=1$, and from (6.17) we get

$$
\gamma_{1} \leq F^{*}\left(x^{*}\right)
$$

Since $y_{1}^{*}=0,(6.11)$ yields $y_{0}^{*}+y_{M}^{*}=0$. Thus, condition (6.14) takes the form

$$
\varphi(y)<t \quad \Rightarrow \quad-\left\langle y_{M}^{*}, y\right\rangle+t \geq \gamma_{0} .
$$


For any $y \in Y$ and any $\varepsilon>0$ let us choose $t=\varphi(y)+\varepsilon$. Then it follows from (6.19) that $-\left\langle y_{M}^{*}, y\right\rangle+\varphi(y)+\varepsilon \geq \gamma_{0}$ for all $y$, whence $\varphi^{*}\left(y_{M}^{*}\right) \leq-\gamma_{0}+\varepsilon$. Since $\varepsilon>0$ can be made arbitrary small, we obtain

$$
\varphi^{*}\left(y_{M}^{*}\right) \leq-\gamma_{0} .
$$

From (6.12), the third condition in (6.16), and (6.18), (6.20) we deduce

$$
\left\langle y_{M}^{*}, y_{0}\right\rangle+F^{*}\left(x^{*}\right)-\varphi^{*}\left(y_{M}^{*}\right) \geq 0 .
$$

Set $y^{*}=y_{M}^{*}$. Then $y^{*} \in \operatorname{dom} \varphi^{*}, x^{*}=A^{*} y^{*}$, and (6.5) is fulfilled.

\section{REFERENCES}

[1] J.-P. Aubin, Contingent derivatives of set-valued maps and existence of solutions to nonlinear inclusions and differential inclusions, In: Nachbin L. (ed.) Mathematical anAlysis AND applications, Part A, Academic Press, New York, 1981, pp. 160-229.

[2] J.-P. Aubin and H. Frankowska, Set-Valued Analysis, Birkhäuser, Berlin, 1990.

[3] J.F. Bonnans and A. Hermant, Second-order analysis for optimal control problems with pure state constraints and mixed control-state constraints, Ann. Inst. H. Poincaré Anal. Non Linéaire, 26 (2009), pp. 561-598

[4] A. Cernea and H. Frankowska, A connection between the maximum principle and dynamic programming for constrained control problems, SIAM J. Control Optim., 44 (2005), pp. $673-703$.

[5] A.Ya. Dubovitski and A.A. Milyutin, Extremum problems with constraints, Dokl. Akad. Nauk SSSR, 149 (4) (1963), pp. 759-762; Soviet Math. Dokl., 4 (1963), pp. 452-455.

[6] A.YA. Dubovitski And A.A. Milyutin, Extremum problems in the presence of restrictions, Zh. Vychisl. Mat. Mat. Fiz., USSR Comput. Math. and Math. Phys., 5 (1965), pp. 1-80.

[7] H. Frankowska, The maximum principle for an optimal solution to a differential inclusion with end point constraints, SIAM J. Control Optim., 25 (1987), pp. 145-157.

[8] H. Frankowska, D. Hoenener and D. Tonon, A second-order maximum principle in optimal control under state constraints, Serdica Mathematical Journal, 39 (2013), pp. 233-270.

[9] H. Frankowska and N. OsmolovskiI, Second-order necessary optimality conditions for the Mayer problem subject to a general control constraint, in ANALYSis AND GeOMETRY iN Control theory and its Applications, Springer INdAM Ser., 11, Springer, Cham, 2015, pp. 171-207.

[10] H. Frankowska and N. Osmolovskit, Second-order necessary conditions for a strong local minimum in a control problem with general control constraints, Applied Mathematics and Optimization, https://doi.org/10.1007/s00245-017-9461-x.

[11] R.V. GAMkRELIDZE, Optimal processes with bounded phase coordinates, Izv. Akad. Nauk, USSR Sec. Mat., 24 (1960), pp. 315-356.

[12] R.F. Hartl, S.P. Sethi And R.G. Vickson, A survey of the maximum principles for optimal control problems with state constraints SIAM Review, 37 (1995), pp. 181-218.

[13] D. Hoenener, Variational approach to second-order optimality conditions for control problems with pure state constraints, SIAM J. Control Optim., 50 (2012), pp. 1139-1173.

[14] D. Hoenener, Feasible perturbations of control systems with pure state constraints and applications to second-order optimality conditions, Appl. Math. Optim., 68 (2013), pp. 219-253.

[15] A.D. Ioffe and V.M. Tichomirov, Theory of Extremal Problems, North-Holland, Amsterdam 1979.

[16] E.S. Levitin, A.A. Milyutin And N.P. Osmolovskir, On conditions for a local minimum in a problem with constraints [in Russian], in: B.S. Mitjagin (Ed.), Mathematical economics and functional analysis, Nauka, Moscow 1974, pp. 139-202.

[17] E.S. Levitin, A.A. Milyutin And N.P. Osmolovskis, Conditions of high order for a local minimum in problems with constraints, Russian Math. Surveys, 33 (1978), pp. 97-168.

[18] K. Malanowski, H. Maurer and S. Pickenhain, Second-order sufficient conditions for stateconstrained optimal control problems, J. Optim. Theory Appl., 123 (2004), pp. 595-617.

[19] A.A. Milyutin, On a certain family of optimal control problems with phase constraint, J. Math. Sc., 100 (2000), 2564-2571.

[20] A.A. Milyutin, A.V. Dmitruk and N.P. Osmolovskit, Maximum principle in optimal CONTROL [in Russian], Moscow State University, Faculty of Mechanics and Mathematics, Moscow, 2004. 
[21] Z. Pales And V. Zeidan, First- and second-order optimality conditions for a strong local minimum in control problems with pure state constraints, Nonlinear Anal., 67 (2007), pp. 2506-2526.

[22] I.B. Russak, Second order necessary, conditions for problems with state inequality constraints, SIAM J. Control Optim., 13 (1975), 372-388.

[23] R.B. Vinter, Optimal Control, Birkhäuser Boston, Inc., Boston, MA, 2000. 